\title{
The First Planetary Microlensing Event with Two Microlensed Source Stars
}

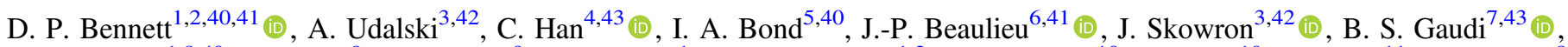

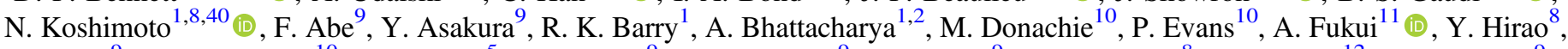
Y. Itow ${ }^{9}$ (1) M. C. A. Li $^{10}$, C. H. Ling ${ }^{5}$, K. Masuda ${ }^{9}$, Y. Matsubara ${ }^{9}$, Y. Muraki ${ }^{9}$, M. Nagakane ${ }^{8}$, K. Ohnishi ${ }^{12}$, H. Oyokawa ${ }^{9}$, C. Ranc ${ }^{1}$, N. J. Rattenbury ${ }^{10}$ (i), M. M. Rosenthal ${ }^{13}$, To. Saito ${ }^{14}$, A. Sharan ${ }^{10}$, D. J. Sullivan ${ }^{15}$, T. Sumi ${ }^{8}$, D. Suzuki ${ }^{16}$ (1), P. J. Tristram ${ }^{17}$, A. Yonehara ${ }^{9}$

(The MOA Collaboration),

\section{K. Szymański ${ }^{3}$, R. Poleski ${ }^{3,7}$, I. Soszyński ${ }^{3}$, K. Ulaczyk ${ }^{3}$, Ł. Wyrzykowski ${ }^{3}$}

(The OGLE Collaboration),

D. DePoy ${ }^{18}$, A. Gould ${ }^{7,19,20}$, R. W. Pogge ${ }^{7}$, J. C. Yee ${ }^{21}$ (1)

(The $\mu$ FUN Collaboration),

and

M. D. Albrow ${ }^{22}$ (D) E. Bachelet ${ }^{23}$ (D), V. Batista ${ }^{6}$ (D), R. Bowens-Rubin ${ }^{24}$, S. Brillant ${ }^{25}$, J. A. R. Caldwell ${ }^{26}$ (D) A. Cole ${ }^{27}$, C. Coutures ${ }^{6}$, S. Dieters ${ }^{27}$, D. Dominis Prester ${ }^{28}$, J. Donatowicz ${ }^{29}$, P. Fouqué ${ }^{30,31}$, K. Horne ${ }^{32}$ (D), M. Hundertmark ${ }^{32,33}$ (iD, N. Kains ${ }^{34}$ (D), S. R. Kane ${ }^{35}$ (D), J.-B. Marquette ${ }^{6}$, J. Menzies ${ }^{36}$, K. R. Pollard ${ }^{22}$, C. Ranc ${ }^{1}$, K. C. Sahu ${ }^{34}$ (D), J. Wambsganss ${ }^{37}$ (iD, A. Williams ${ }^{38,39}$, and M. Zub ${ }^{37}$

(The PLANET Collaboration)

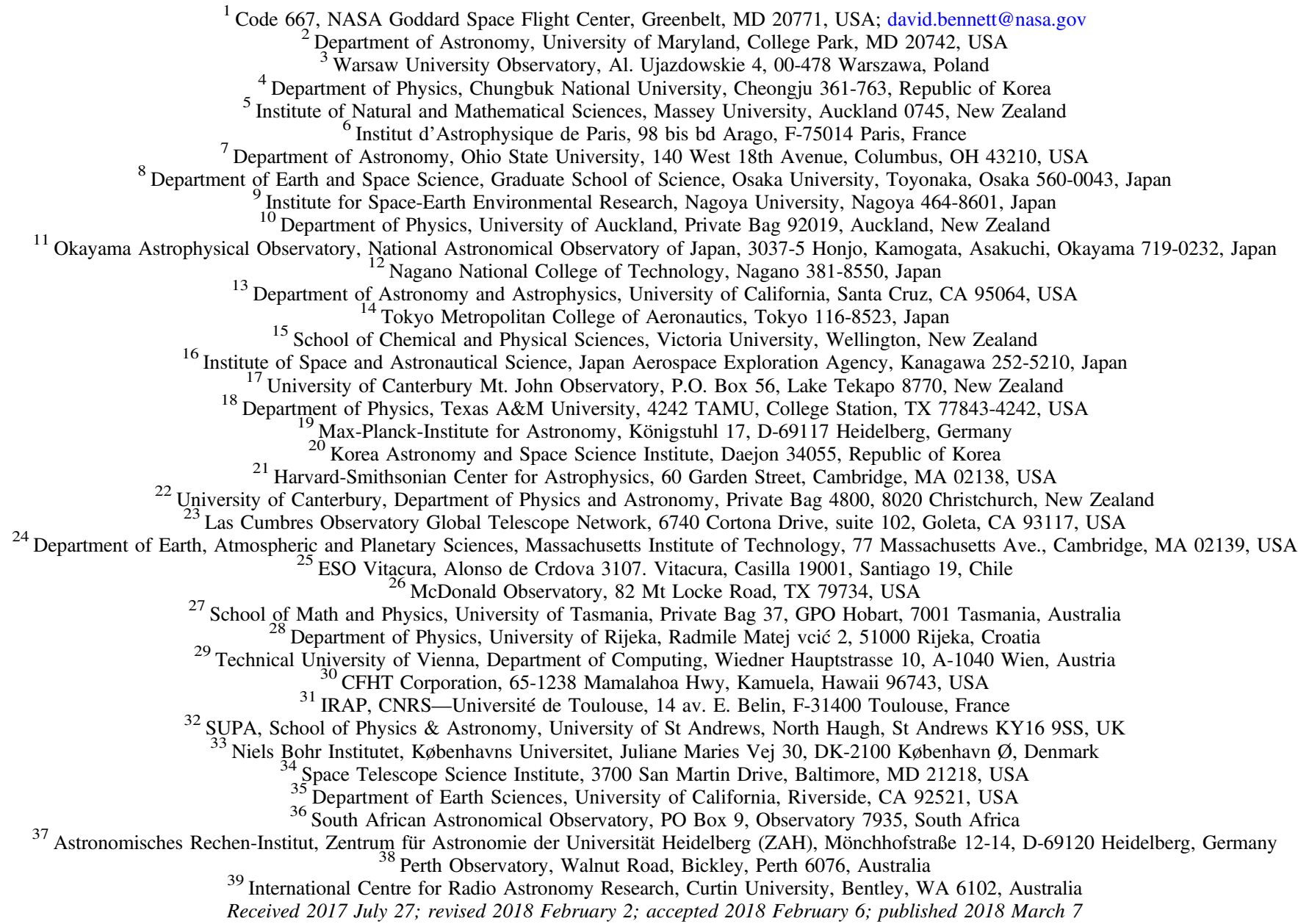

\footnotetext{
${ }^{40}$ MOA Collaboration.

${ }^{41}$ PLANET Collaboration.

42 OGLE Collaboration.

$43 \mu$ FUN Collaboration.
} 


\begin{abstract}
We present the analysis of the microlensing event MOA-2010-BLG-117, and show that the light curve can only be explained by the gravitational lensing of a binary source star system by a star with a Jupiter-mass ratio planet. It was necessary to modify standard microlensing modeling methods to find the correct light curve solution for this binary source, binary-lens event. We are able to measure a strong microlensing parallax signal, which yields the masses of the host star, $M_{*}=0.58 \pm 0.11 M_{\odot}$, and planet, $m_{p}=0.54 \pm 0.10 M_{\mathrm{Jup}}$, at a projected star-planet separation of $a_{\perp}=2.42 \pm 0.26 \mathrm{au}$, corresponding to a semimajor axis of $a=2.9_{-0.6}^{+1.6}$ au. Thus, the system resembles a half-scale model of the Sun-Jupiter system with a half-Jupiter0mass planet orbiting a half-solar-mass star at very roughly half of Jupiter's orbital distance from the Sun. The source stars are slightly evolved, and by requiring them to lie on the same isochrone, we can constrain the source to lie in the near side of the bulge at a distance of $D_{S}=6.9 \pm 0.7 \mathrm{kpc}$, which implies a distance to the planetary lens system of $D_{L}=3.5 \pm 0.4 \mathrm{kpc}$. The ability to model unusual planetary microlensing events, like this one, will be necessary to extract precise statistical information from the planned large exoplanet microlensing surveys, such as the WFIRST microlensing survey.
\end{abstract}

Key words: gravitational lensing: micro - planetary systems

\section{Introduction}

Gravitational microlensing has a unique niche among planet discovery methods (Bennett 2008; Gaudi 2012) because of its sensitivity to planets with masses extending to below an Earth mass (Bennett \& Rhie 1996) orbiting beyond the snow line (Mao \& Paczyński 1991; Gould \& Loeb 1992), where planet formation is thought to be the most efficient, according to the leading core accretion theory of planet formation (Lissauer 1993; Pollack et al. 1996). While radial velocity and planetary transit surveys (Ida \& Lin 2005; Kennedy et al. 2006; Lecar et al. 2006; Kennedy \& Kenyon 2008; Thommes et al. 2008; Wright \& Gaudi 2013; Twicken et al. 2016) have found hundreds and thousands of planets, respectively, these methods have much higher sensitivity to planets that orbit very close to their host stars. Their sensitivity to planets like those in our own solar system is quite limited. Our knowledge of these wide-orbit planets extending down to low masses depends on the results of microlensing surveys (Gould et al. 2010b; Cassan et al. 2012; Suzuki et al. 2016). This is the main reason for the selection of the space-based exoplanet microlensing survey (Bennett \& Rhie 2002) to be a part of the WFIRST mission (Spergel et al. 2015), which was the top-rated large space mission in the 2010 New Worlds, New Horizons decadal survey.

Like the Kepler transit survey (Borucki et al. 2011), the WFIRST exoplanet microlensing survey will primarily be a statistical survey with thousands of expected exoplanet discoveries. However, a large number of planet discoveries does not automatically translate into good statistics if a large fraction of the planet candidates do not allow precise interpretations (Burke et al. 2015; Mullally et al. 2016). Fortunately, the microlensing method predicts a relatively small number of low signal-to-noise planet candidates (Gould et al. 2004) compared to the transit method. Nevertheless, microlensing does have the potential problem of microlensing events that defy interpretation, and these could also add to the statistical uncertainty in the properties of the exoplanet population that can be studied by microlensing.

In the past two years, the analysis of several complicated microlensing events potentially involving planets has been completed. The lens system for OGLE-2007-BLG-349 was revealed to be a circumbinary planet, rather than a two-planet system with a single host star (Bennett et al. 2016). This removed a significant uncertainty from the Gould et al. (2010b), Cassan et al. (2012), and Suzuki et al. (2016) statistical analyses, which included this event. (If the twoplanet model for OGLE-2007-BLG-349 had been correct, the second planet would have been the lowest mass ratio planet discovered by microlensing.) Another complicated event was OGLE-2013-BLG-0723, which was originally claimed to be a planet in a binary star system that was unusually close to the Sun for a microlensing event (Udalski et al. 2015a). This small distance to the lens system was due to a large microlensing parallax signal. However, a more careful analysis of the data (Han et al. 2016) indicated that the light curve was better explained by a binary star system without a planet and a much smaller microlensing parallax signal. Most recently, Han et al. (2017) analyzed a planet in a binary star system and found a somewhat ambiguous result, with solutions consisting of a planet and stellar (or brown dwarf) hosts with mass ratios ranging from 0.95 to 0.03 .

In this paper, we present the analysis of the microlensing event MOA-2010-BLG-117, an event that has eluded precise interpretation for several years after it was observed and identified as a planetary microlensing event. It has a strong planetary signal, so it must be included in the statistical analysis of MOA data (Suzuki et al. 2016). In fact, the basic character of the light curve was obvious by inspection to many of the authors of this paper. There was a clear planetary signal due to the crossing of two minor image caustics, but detailed models did not provide a good fit. The region between these two minor image caustics is an area of strong demagnification because the minor image is largely destroyed in this region, but the magnification between MOA-2010-BLG-117 was simply too large. It could only be fit with the addition of a fourth body to increase the magnification between the minor image caustics. This fourth body could be a second source star that would not pass between the minor image caustics and would therefore not suffer the demagnification experienced by the first source. Or the fourth body could be a third lens that could provide additional magnification between the minor image caustics. We found that the only viable triple-lens systems were ones the with two stars orbited by one planet, and that two-planet models could not match the observed light curve. The early modeling could not decide between the binary source and circumbinary planet possibilities.

This paper is organized as follows. In Section 2, we describe the light curve data, photometry, and real-time modeling that influenced some of the data collection strategy. In Section 3, we describe the systematic light curve modeling of the final data set, which shows that the binary source model must be 
correct. We also show that we can constrain the distance to the source by requiring that the two source stars have magnitudes and colors that lie on the same isochrone. We describe the photometric calibration and the determination of the primary source star radius in Section 4, and then we derive the lens system properties in Section 5. In Section 6, we consider high angular resolution adaptive optics (AO) observations of the MOA-2010-BLG-117 target, and we present a proper motion measurement of the MOA-2010-BLG-117 target that indicates that the source star system lies in the Galactic bulge. Our conclusions are presented in Section 7.

\section{Light Curve Data, Photometry and Real Time Modeling}

The microlensing event MOA-2010-BLG-117, at R.A. = 18:07:49.67, decl. $=-25: 20: 40.7$, and Galactic coordinates $(l, b)=(5.5875,-2.4680)$, was identified and announced as a microlensing candidate by the Microlensing Observations in Astrophysics (MOA) Collaboration Alert system (Bond et al. 2001) on 2010 April 7. The MOA team subsequently identified the light curve as anomalous at UT 10:19 am, 2010 August 2, and this announcement triggered follow-up observations by the Probing Lensing Anomalies NETwork (PLANET) and the MICROlensing Follow-up Network ( $\mu$ FUN). The PLANET group observed this event using the $1.0 \mathrm{~m}$ telescope at the South African Astronomical Observatory (SAAO), and the $\mu$ FUN group used the 1.3 SMARTS telescope at the Cerro Tololo Interamerican Observatory (CTIO). The Optical Gravitational Lensing Experiment (OGLE) Collaboration had just updated to their wide field of view OGLE-4 system (Udalski et al. 2015b), and their Early Warning System (EWS) was not yet in operation with the new camera (Udalski et al. 1994). So, the OGLE photometry was not produced automatically by the EWS system, but once it became clear that this event had a likely planetary signal, OGLE began to reduce and circulate their data.

After some systematic trends with airmass were removed from the MOA data and the OGLE data was released, it became clear by inspection that the light curve of this event resembled the case of a source that crossed the region of the triangular minor image caustics, hitting both caustics. This configuration is somewhat similar to that of OGLE-2007-BLG-368 (Sumi et al. 2010) and MOA-2009-BLG-266 (Muraki et al. 2011), except that the source for OGLE-2007-BLG-368 only crossed one of the minor image caustics and the source for MOA-2009BLG-266 was almost as large as the minor image caustics. However, attempts to model this event did not yield good fits with this geometry.

The problem with this minor image caustic-crossing model is that the magnification deficit between the two caustic (or cusp) crossings at $t=5402$ and 5411 is too small. (Note that $t \equiv$ HJD - 2450000). This is evident in Figure 1, which shows the best-fit binary-lens light curve for MOA-2010-BLG-2010. This light curve has the obvious problem that the magnification between the two caustic/cusp features is higher than the model can accommodate. In fact, the problem is more severe than this figure indicates. In order to minimize this discrepancy between the model and the data, the event is driven to have a very bright source, so that the minor image will be kept at relatively low magnification, which reduces the magnification deficit between the two caustic/cusp features. However, in this case, the source brightness is driven to be $1.5 \times$ brighter than the apparent source star in the OGLE images. This means that negative blending is required, since a negative "blend flux" must be added to the source flux to achieve the relatively faint "star" seen in the unmagnified images. Negative blending is quite possible at low levels due to the variations in the apparent "sky" background due to unresolved stars, but in this case, the level of negative blending is too large for such a physical explanation. So, it implies that this model is likely to be incorrect.

Because of these difficulties with the minor image perturbation model and unrelated difficulties with the real-time photometry, early attempts at modeling this event predicted that the relatively bright, well-observed feature at $t \approx 5411$ was the interior of a caustic entrance, where the caustic crossing itself was not observed. But, a subsequent caustic exit never occurred. This made it clear that some version of a planetary minor caustic-crossing event was correct, but that an additional lens or source was needed to explain the higher-than-expected brightness between the two caustic/cusp crossings. This possibility was recognized relatively early after the discovery of the light curve anomaly, so we obtained more frequent CTIO $V$-band observations than usual in the hopes that they might help reveal a color difference between the two sources of a binary source model.

It was necessary to wait until mid-2011 before the magnification was back at baseline because of the long duration of this microlensing event. After that, the OGLE Collaboration provided optimal centroid photometry using the OGLE difference imaging pipeline (Udalski 2003). Photometry of the MOA data was performed with the MOA pipeline (Bond et al. 2001), which also employs the difference imaging method (Tomaney \& Crotts 1996). The PLANET collaboration's SAAO data were reduced with a version of the Pysis difference imaging code (Albrow et al. 2009), and the CTIO data were reduced with DoPHOT (Schechter et al. 1993). The final data set consists of 4966 MOA observations in the custom MOARed passband (roughly equivalent to the sum of Cousins $R+I$ ), 398 and 48 OGLE observations in the $I$ and $V$ bands, respectively, $150 \mathrm{I}$-band and $88 \mathrm{~V}$-band observations from the SMARTS telescope in CTIO, 119 I-band observations from SAAO, and $10 \mathrm{~K}$-band observations from the VVV survey (Minniti et al. 2010) using the VISTA telescope at Paranal, which happened to be doing a low-cadence survey of the Galactic bulge in 2010 .

\section{Light Curve Models}

Our light curve modeling was done using the image-centered ray-shooting method (Bennett \& Rhie 1996; Bennett 2010), supplemented with the hexadecapole approximation (Gould 2008; Pejcha \& Heyrovský 2009) that is employed as a test for accuracy. For triple-lens modeling, we used the code developed for OGLE-2006-BLG-109 (Bennett et al. 2010) and OGLE2007-BLG-349 (Bennett et al. 2016). Triple-lens models have some parameters in common with single- and binary-lens models. These are the Einstein radius crossing time, $t_{E}$, and the time, $t_{0}$, and distance, $u_{0}$, of closest approach between the lens' center of mass and the source star. For a binary lens, there is also the mass ratio of the secondary to the primary lens, $q$, the angle between the lens axis and the source trajectory, $\theta$, and the separation between the lens masses, $s$.

The length parameters, $u_{0}$ and $s$, are normalized by the Einstein radius of this total system mass, $R_{E}=$ $\sqrt{\left(4 G M / c^{2}\right) D_{S} x(1-x)}$, where $x=D_{L} / D_{S}$, and $D_{L}$ and $D_{S}$ are the lens and source distances, respectively. ( $G$ and $c$ are 


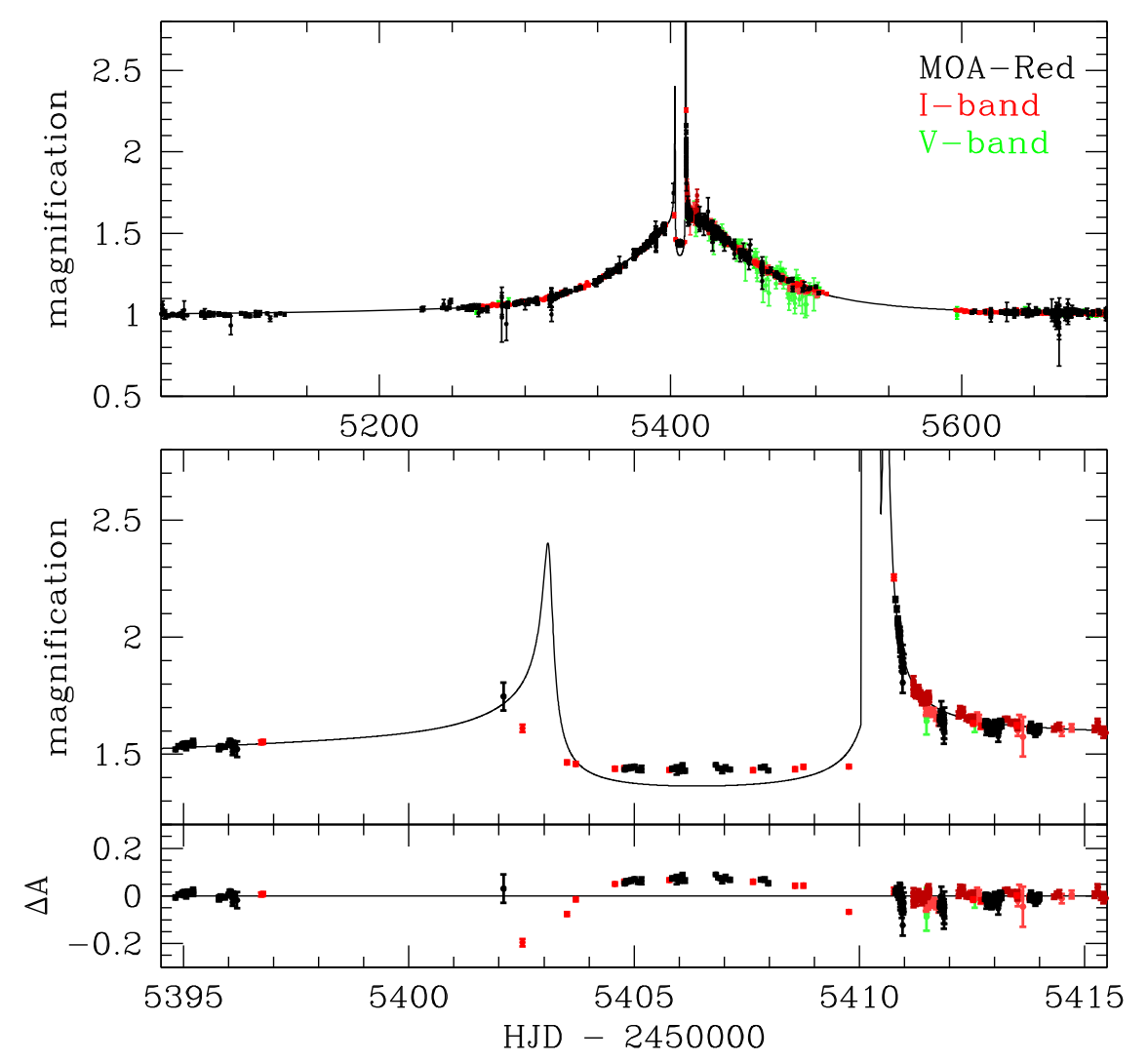

Figure 1. Best binary-lens model for the MOA-2010-BLG-117 light curve. MOA-Red band data are shown in black. I-band data from OGLE, CTIO, and SAAO are shown in red, light red, and dark red, respectively, while the OGLE and CTIO $V$-band data are shown in green and light green.

the gravitational constant and speed of light, as usual.) For triple-lens models, there are additional separation, mass ratio, and angle to describe the position and mass ratio of the third lens, but we will not explore these models in detail in this paper.

For every passband, there are two parameters to describe the unlensed source brightness and the combined brightness of any unlensed "blend" stars that are superimposed on the source. Such "blend" stars are quite common because microlensing is only seen if the lens-source alignment is $\lesssim \theta_{E} \sim 1$ mas, while stars are unresolved in ground-based images if their separation is $\lesssim 1^{\prime \prime}$. However, with ground-based seeing, the background contains many unresolved stars, and this makes the background uneven. As a result, it is possible to have realistic cases of "negative blending" if the "negative" brightness of the blend is consistent with the fluctuations in the unresolved stellar background. Artificial negative blending can occur with difference imaging photometry, which does not attempt to identify a source star in the reference image, but this is just an artifact of the photometry method. In any case, these source and blend fluxes are treated differently from the other parameters because the observed brightness has a linear dependence on them, so for each set of nonlinear parameters, we can find the source and blend fluxes that minimize the $\chi^{2}$ exactly, using standard linear algebra methods (Rhie et al. 1999).

For the binary source models for MOA-2010-BLG-117, we add a second source to the binary-lens model, allowing for a different brightness and color for the second source. The second source has its own $t_{0}$ and $u_{0}$ values, which we denote as $t_{0 s 2}$ and $u_{0 s 2}$. If the two source stars have exactly the same velocity, then the $t_{E}$ and $\theta$ values for the two sources would also be the same, but due to orbital motion, the $t_{E}$ and $\theta$ values are slightly different. However, the orbital motion of the source stars is much smaller than the orbital motion of the source star system in the Galaxy, so we use parameters to describe the difference in the $t_{E}$ and $\theta$ values. The parameters we use are $d t_{E s 2}=t_{E s 2}-t_{E s 1}$ and $d \theta_{s 2}=\theta_{s 2}-\theta_{s 1}$, where $t_{E}=t_{E s 1}$ and $\theta=\theta_{s 1}$.

Our initial attempts to model this event favored the circumbinary models, and the model shown in Figure 2 was the best fit. However, there are several problems with this model. First, although the data are sparse, the model does not provide a good fit to the first cusp approach at $t=5402-5403$. However, there is a more serious problem with this model that is demonstrated by Figure 3, which shows how the orbital motion of the binary host stars affects the caustic configuration. The central caustic rotates quite rapidly, such that the angle between the direction of the right-pointing cusp and the source position remains nearly constant throughout the interval between the cusp crossings. This is apparently necessary to avoid having a local light curve peak in the middle of the long minimum at $5403.5<t<5410$ at a location where the cusp would be pointing directly at the source. With the rapid orbital motion implied by this model, the source can remain at the same angle with respect to the cusp direction throughout the passage of this light curve minimum.

The rapid orbital motion presents a problem, however. The probability of lensing by two stars that are not bound to each other is quite small $\left(\sim 10^{-12}\right)$, so we can assume that the two lens stars are bound. If so, then their relative velocity cannot be above the escape velocity of the system. As a result, the high relative velocity implies that the lens must be close to either the 


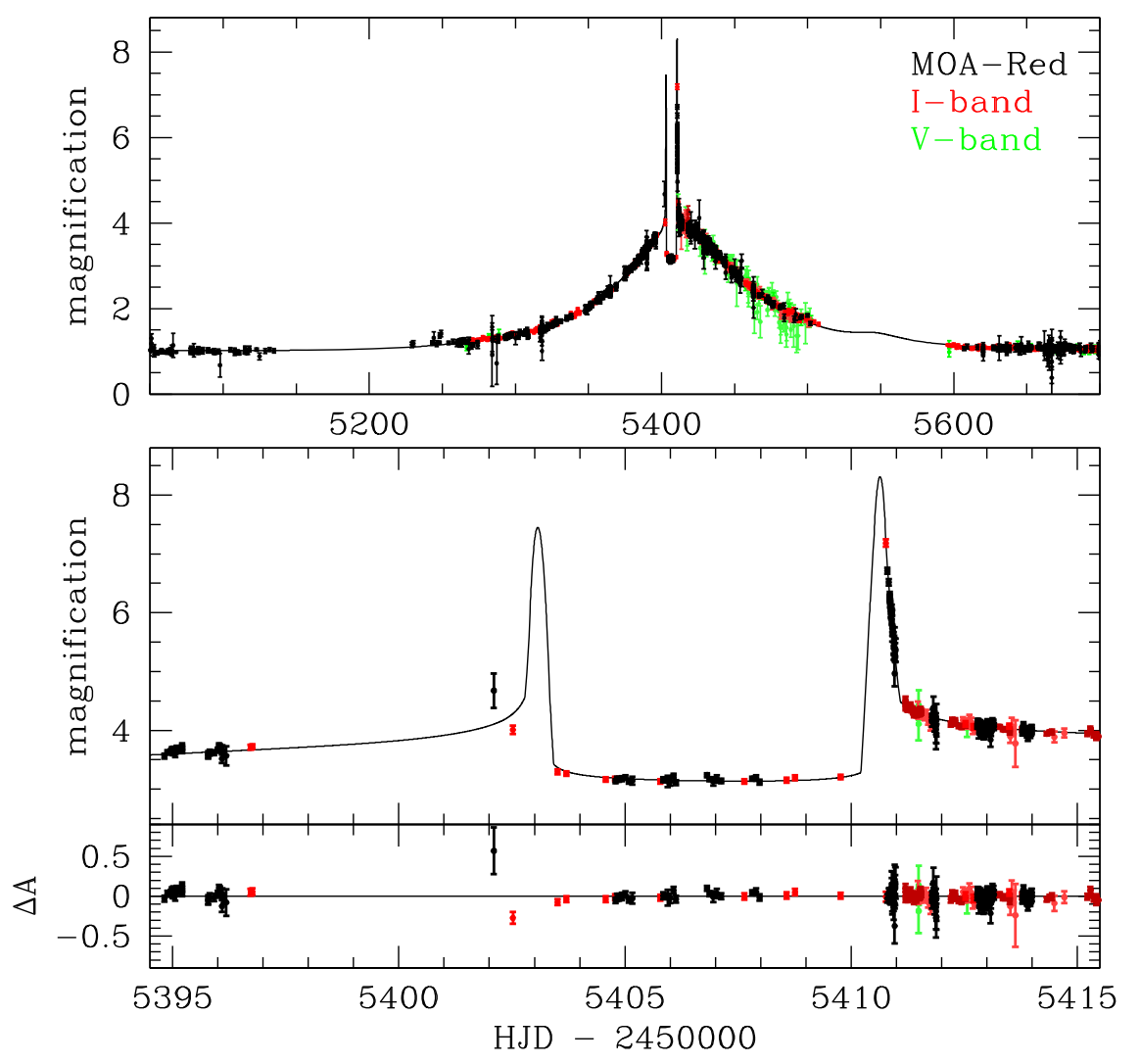

Figure 2. Best circumbinary-lens model for the MOA-2010-BLG-117 light curve. MOA-Red band data are shown in black. I-band data from OGLE, CTIO, and SAAO are shown in red, light red, and dark red, respectively, while the OGLE and CTIO $V$-band data are shown in green and light green.

lens or the observer, because both of these possibilities allow higher lens orbital velocities when measured in units of Einstein radii per unit time. With the angular source radius, $\theta_{*}$, derived below in Section 4, we can derive the angular Einstein radius, $\theta_{E}=\theta_{*} t_{E} / t_{*}$, and this yields the following relation (Bennett 2008; Gaudi 2012)

$$
\begin{aligned}
M_{L}= & \frac{c^{2}}{4 G} \theta_{E}^{2} \frac{D_{S} D_{L}}{D_{S}-D_{L}}=0.9823 M_{\odot} \\
& \times\left(\frac{\theta_{E}}{1 \mathrm{mas}}\right)^{2}\left(\frac{x}{1-x}\right)\left(\frac{D_{S}}{8 \mathrm{kpc}}\right),
\end{aligned}
$$

where $x=D_{L} / D_{S}$ and $\theta_{E} \sim 0.8$ mas for this event. This allows us to determine the lens system mass and convert the measured transverse separation and velocity to physical units at every possible distance for the lens. This exercise tells us that the two stars would be unbound for $0.93 \mathrm{kpc}<D_{L}<7.5 \mathrm{kpc}$ and $0.05 M_{\odot}<M_{L}<26 M_{\odot}$. However, the microlensing parallax parameters for this model imply a lens system mass of $M_{L}=0.218 M_{\odot}$. We can conclude that the lens orbital velocity parameters are too large for a physically reasonable model, and so the binary source model is favored.

Although the best circumbinary model implied unphysical parameters, in our initial modeling, the best circumbinary model had a better $\chi^{2}$ than the best binary source models that we found, by $\Delta \chi^{2}>130$. However, the best binary source models from our first round of fitting had an unphysical feature as well. As with the models with a single source, we had been considering the source brightnesses in each passband as independent parameters. But, this allowed the models to move into unphysical regions of the parameter space, in which the flux ratio between the two sources was very different for passbands that were nearly identical, like the OGLE, CTIO, and SAAO $I$ bands. In order to avoid these unphysical models, we have modified our modeling code to fix the source flux ratio to be the same for each of the I-band data sets and each of the $V$-band data sets. The flux ratio of source 2 to source 1 is given by the parameters $f_{s 2 V}$ and $f_{s 2 I}$ in the $V$ and $I$ bands, respectively. Source 1 is defined to be the source that crosses the planetary caustics. For the MOA-Red band, we do not use a independent flux ratio parameter. Instead, we derive the MOARed band flux ratio parameter from the $I$ - and $V$-band parameters, $f_{s 2 R m}=f_{s 2 I}^{0.837} f_{s 2 V}^{0.163}$. This follows from the color transformation that we have derived from the bright stars in this field (Gould et al. 2010a; Bennett et al. 2012),

$$
R_{\text {moa }}-I_{\mathrm{O} 4}=0.1630\left(V_{\mathrm{O} 4}-I_{\mathrm{O} 4}\right)+\text { const, }
$$

where $V_{\mathrm{O} 4}$ and $I_{\mathrm{O} 4}$ refer to the OGLE-IV $V$-band and $I$-band magnitudes that have been used for the OGLE light curve data. Note that these restrictions are more restrictive than those used for some previous non-planetary binary source events that only constrained data sets using the same passband with the same flux ratio (Hwang et al. 2013; Jung et al. 2017).

With these limitations on the source brightness ratios, we found that the binary source models quickly converged to a solution that was better than the previous best binary source model by $\Delta \chi^{2} \sim 200$. It was also better than the best circumbinary model by $\Delta \chi^{2}=68.9$, even though we allowed some of the parameters of the best circumbinary model to take unphysical values. 


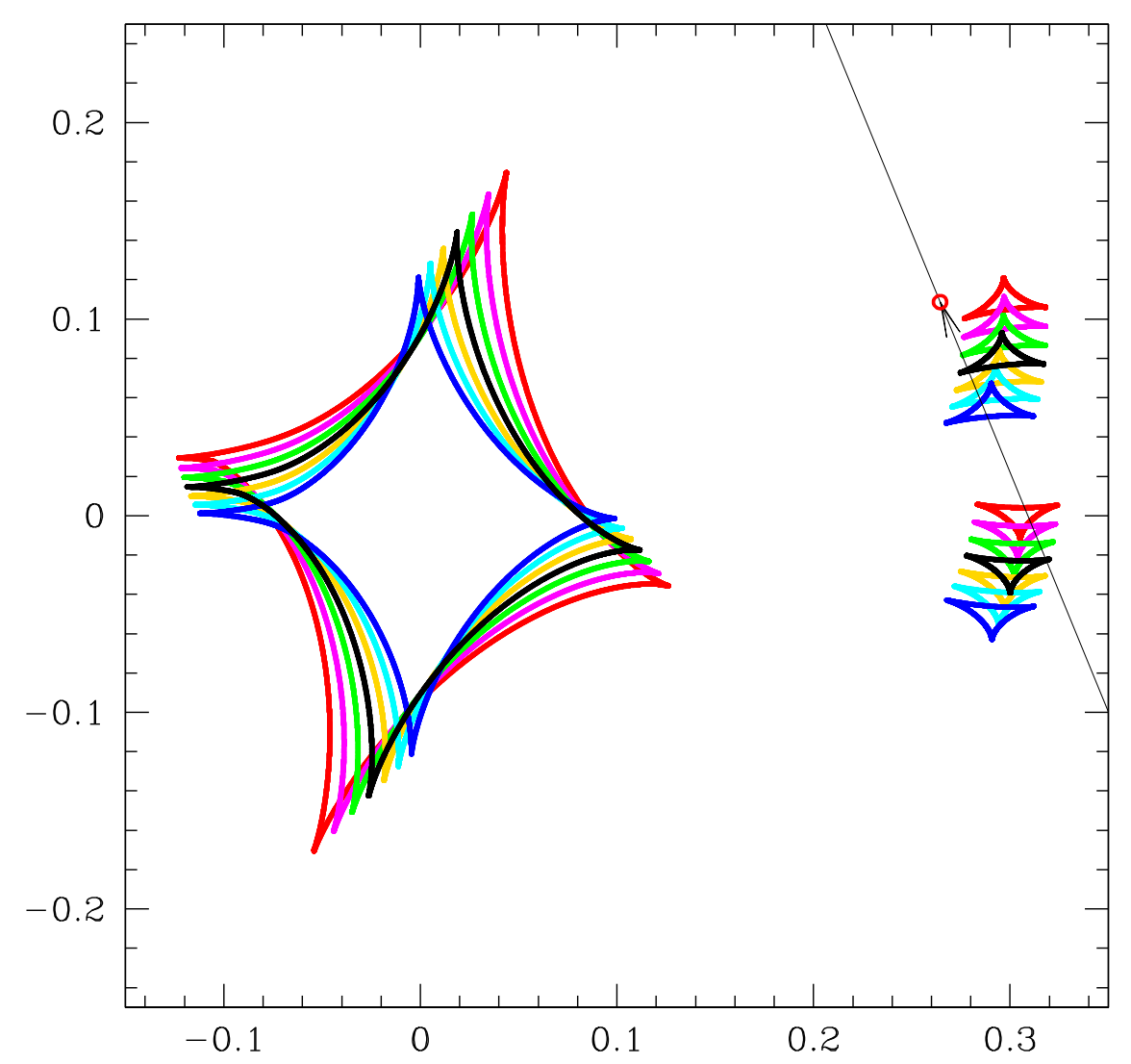

Figure 3. Caustic configuration, shown at an interval of two days for the best circumbinary-lens model for the MOA-2010-BLG-117 light curve. The caustics are shown at $t=5401,5403,5405,5407,5409,5411$, and 5413 in red, magenta, green, black, gold, cyan, and blue, respectively. The source trajectory is given by the black line with the red circle indicating the source size.

The best-fit light curve model is shown in Figure 4, with the parameters listed in the third column of Table 1. (The best-fit solution with $u_{0}<0$ is listed in the fourth column.) Because the sources have different colors, the light curves in the different passbands are different. The green, red, and black curves represent the model light curves in the $V, I$, and $R_{\text {moa }}$ passbands, respectively. The data are plotted with a similar color scheme. We use green and light green for the OGLE and CTIO $V$-band data, black for the $R_{\text {moa }}$ data, and dark red, red, and light red for the SAAO, OGLE, and $\mu$ FUN $I$-band data, respectively. The caustic configuration for the best-fit model is shown in Figure 5. We define the source that crosses the planetary caustic to be source number 1 and the other to be source 2. Although both sources have similar $\left|u_{0}\right| \sim 0.3$ and $\left|u_{0 s 2}\right| \sim 0.3$ values, we know that only one source comes close to the planetary caustics since we see no evidence of a second encounter of the planetary caustics. This implies that the two sources must pass on different sides of the planetary host star so that the signs of $u_{0}$ and $u_{0 s 2}$ must be different.

The model parameters for the best-fit models with $u_{0}>0$ and $u_{0}<0$ are given in Table 1 . Table 2 gives the Markov Chain Monte Carlo (MCMC) averages for the model parameters. This table also includes some derived parameters of physical interest: the angular Einstein radius, $\theta_{E}$, the microlensing parallax amplitude, $\pi_{E}=\sqrt{\pi_{E, E}^{2}+\pi_{E, N}^{2}}$, and the lens-source relative proper motion, $\mu_{\text {rel,G }}$, in an inertial geocentric frame that moves with the Earth at time $t_{\text {fix }}=5411$ days. The source-lens relative velocities for the two sources should be approximately equal because the orbital velocity of two stars separated by approximately an Einstein radius in the Galactic bulge is typically about an order of magnitude smaller than the orbital velocity of stars in the inner Galaxy. So, we expect the lens-source relative velocity vectors for the two sources to differ by no more than $\sim 10 \%$. However, a $\sim 10 \%$ difference between the $t_{E}$ and $\theta$ values for the two sources will have a significant effect on the light curve shape, so we must include parameters to describe $t_{E}$ and $\theta$ for the second source. We chose the parameters $d t_{E s 2} \equiv t_{E s 2}-t_{E s 1}$, where $t_{E s 1} \equiv t_{E}$, and $t_{E s 2}$ are the $t_{E}$ values for the two sources. The different source trajectory angles are described by $d \theta_{s 2} \equiv \theta_{s 2}-\theta_{s 1}$, where $\theta_{s 1} \equiv \theta$ and $\theta_{s 2}$ are the angles between the source trajectories and the lens axis. We also allow for orbital acceleration of the two source stars. We assume a circular orbit for these stars with an orbital period of $T_{\text {Sorb }}$ and projected velocities at time $t_{\mathrm{fix}}=5411$ days implied by the $d t_{E s 2}$ and $d \theta_{s 2}$ values. These are circular orbits in three dimensions following the parameterization of Bennett et al. (2010).

The orbital velocities in the lens system are also important, but since the planetary features in the light curve are detectable for only $\sim 10$ days, we do not need to include the orbital acceleration of the source. We describe the lens orbital velocities with a rotation of the lens system with angular frequency $\omega$ and a velocity of $\dot{s}$ in the separation direction.

This event has a significant orbital microlensing parallax signal (Gould 1992; Alcock et al. 1995), with a $\chi^{2}$ improvement of $\Delta \chi^{2}=43.93$ with nearly equal contributions from the MOA and OGLE data sets. The microlensing parallax is defined by a two-dimensional vector, $\boldsymbol{\pi}_{E}$, with the north and 


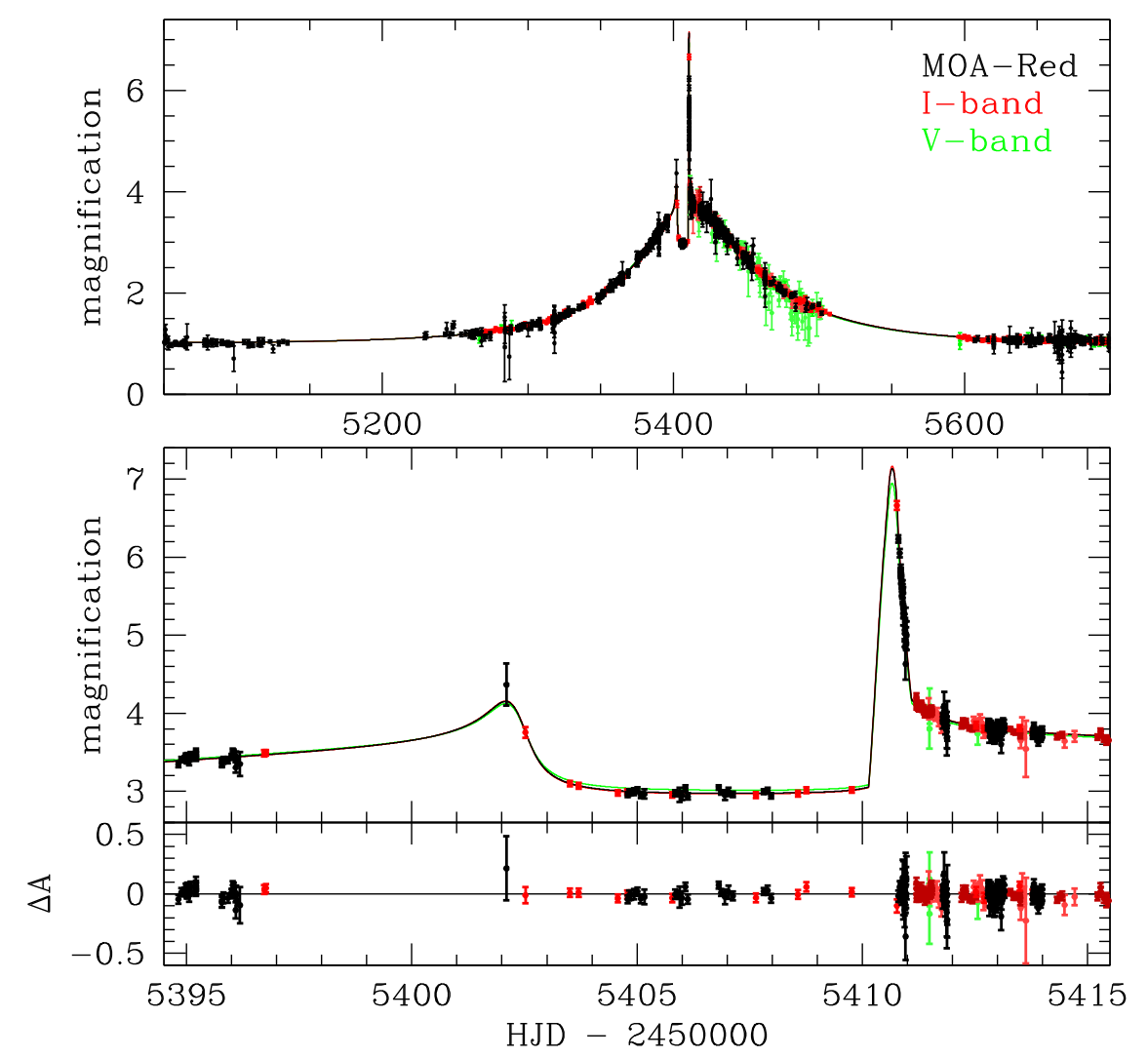

Figure 4. Best binary source model for the MOA-2010-BLG-117 light curve. The $I$-band light curves are plotted in different shades of red, with SAAO as dark red, OGLE as red, and CTIO as light red. The OGLE and CTIO $V$-band light curves are plotted in green and light green, respectively. The MOA-Red band light curve is plotted in black. The model curves for MOA-Red, $I$ band, and $V$ band are plotted in black, red, and green, respectively.

Table 1

Best-fit Model Parameters

\begin{tabular}{llcc}
\hline \hline Parameter & Units & $u_{0}>0$ & $u_{0}<0$ \\
\hline$t_{E}$ & days & 124.57 & 116.64 \\
$t_{0}$ & HJD-2455400 & 19.6850 & 19.8235 \\
$u_{0}$ & $\ldots$ & 0.26539 & -0.29109 \\
$s$ & $\ldots$ & 0.86614 & 0.85531 \\
$\theta$ & radians & 1.95765 & -1.96029 \\
$q$ & $10^{-3}$ & 0.8100 & 0.9451 \\
$t_{*}$ & days & 0.3184 & 0.3511 \\
$\pi_{E, N}$ & $\ldots$ & -0.1759 & 0.1916 \\
$\pi_{E, E}$ & $\ldots$ & -0.0196 & -0.0394 \\
$t_{0 s 2}$ & $\mathrm{HJD}-2455400$ & 0.0189 & 0.0228 \\
$u_{0 s 2}$ & $\ldots$ & -0.27603 & 0.31192 \\
$f_{s 2 I}$ & $\ldots$ & 0.7620 & 0.7631 \\
$f_{s 2 V}$ & $\ldots$ & 0.8583 & 0.8364 \\
$d t_{E s 2}$ & $\ldots$ & -9.16 & -11.80 \\
$d \theta_{s 2}$ & days & 0.32205 & -0.23631 \\
$\omega$ & radians & -0.401 & -1.401 \\
$\dot{s}$ & $10^{-3}$ days $^{-1}$ & -1.862 & -1.607 \\
$1 / T_{S \text { orb }}$ & $10^{-3}$ days $^{-1}$ & 0.5126 & 0.2016 \\
$\theta_{E}$ & $10^{-3}$ days $^{-1}$ & 0.885 & 0.781 \\
fit $\chi^{2}$ & mas & 5744.35 & 5748.56 \\
\hline
\end{tabular}

east components of $\pi_{E, N}$ and $\pi_{E, E}$ in a geocentric coordinate system moving with the velocity of the Earth measured at time $t_{\text {fix }}=5411$ days. The parameter $t_{\text {fix }}$ is also the reference time for the source and lens positions.

We should note that there are upper limits on the relative velocities between the two sources and between the lens star and planet since they must (almost certainly) be gravitationally bound systems. We assume that the source stars each have a solar mass and compare the two-dimensional kinetic energy to the maximum binding energy of the source stars (using their separation on the plane of the sky). Then, following Muraki et al. (2011), we apply a constraint on the $d t_{E s 2}$ and $d \theta_{s 2}$ values. For the lens system, we know the lens mass from the microlensing parallax parameters and the angular Einstein radius, $\theta_{E}$ (Gould 1992; Bennett 2008; Gaudi 2012), and we use this to apply the same constraint. In both cases, the orbital semimajor axis is proportional to $\theta_{E}$.

These lens and source orbital motion constraints are sensitive to the source radius crossing time through $\theta_{E}=t_{E} \theta_{*} / t_{*}$, but the light curve constraint on $t_{*}$ is relatively weak because the caustic crossings are only partially covered. The initial fits to this event with no microlensing parallax, no lens orbital motion, and $d t_{E s 2} \equiv 0$ and $d \theta_{s 2} \equiv 0$ had a large variation in $t_{*}$ values ranging from 0.24 to 0.40 days. When we allowed the $d t_{E s 2}$ and $d \theta_{s 2}$ values to vary, subject only to the constraint on the maximum orbital motion of the source stars, we found that large values of these parameters were preferred. However, the semimajor axis of the orbit of the source stars is proportional to $\theta_{E}=t_{E} \theta_{*} / t_{*}$. Thus, a larger $t_{*}$ implies a smaller $\theta_{E}$ and therefore a smaller semimajor axis. The smaller semimajor axis implies a higher gravitational binding energy, which allows larger lens star velocities, implying larger values for $d t_{E s 2}$ and $d \theta_{s 2}$. Since the data apparently prefer larger values for $d t_{E s 2}$ and $d \theta_{s 2}$, the constraint on $t_{*}$ becomes tighter when we include nonzero values of $d t_{E s 2}$ and $d \theta_{s 2}$, and apply the orbital motion constraint. This can be seen from Figure 6 . Values of $t_{*}<0.26$ days are excluded, and the $2 \sigma$ lower limit on $t_{*}$ is 

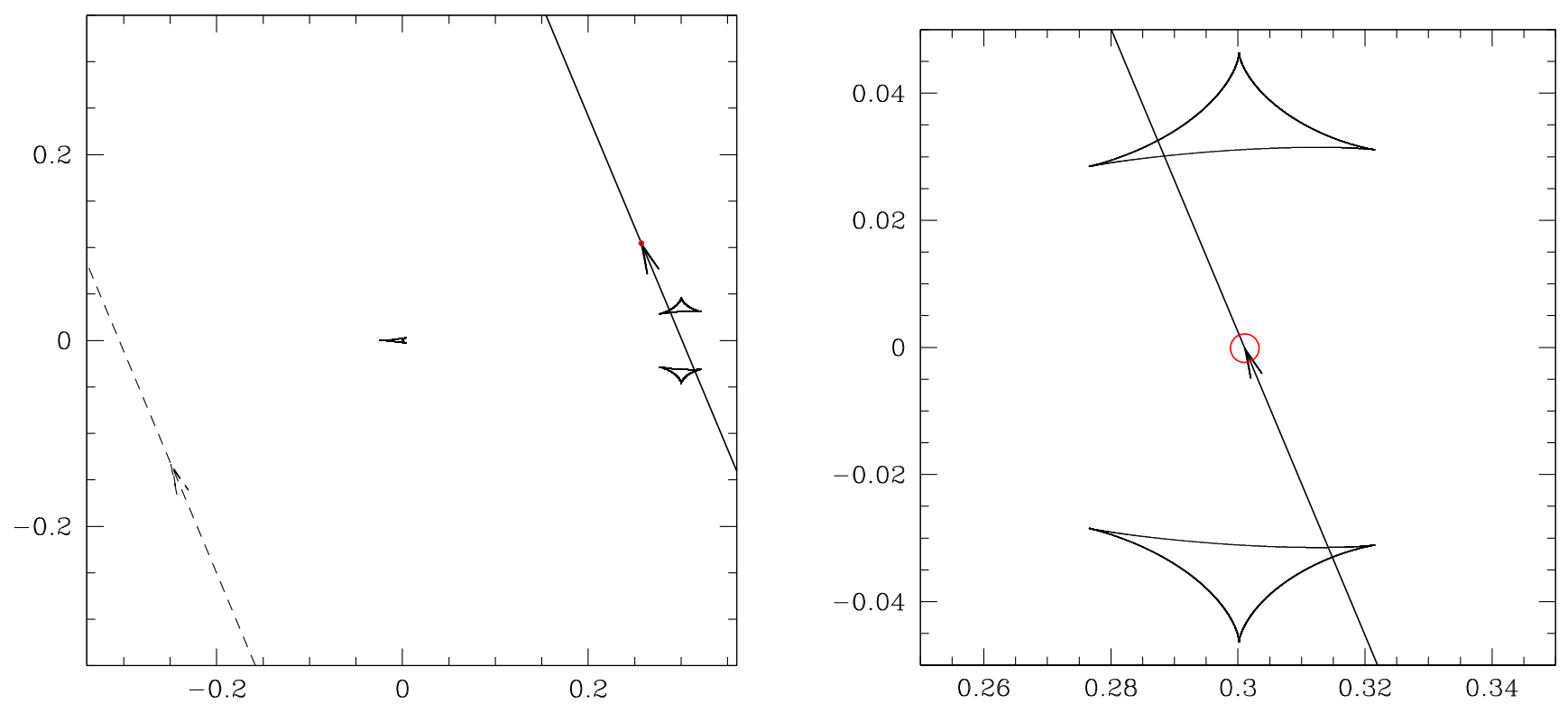

Figure 5. MOA-2010-BLG-117 caustic configuration with the source trajectories shown as the solid and dashed curves for sources 1 and 2, respectively. The arrows give the direction of motion for the sources with respect to the lens system, and the red circle indicates source star 1.

Table 2

MCMC Parameter Distributions

\begin{tabular}{llcc}
\hline \hline Parameter & Units & $u_{0}>0$ & $u_{0}<0$ \\
\hline$t_{E}$ & days & $120.6(5.2)$ & $116.4(4.3)$ \\
$t_{0}$ & HJD -2455400 & $19.80(30)$ & $19.77(28)$ \\
$u_{0}$ & $\ldots$ & $0.279(15)$ & $-0.287(13)$ \\
$s$ & $\ldots$ & $0.8601(60)$ & $0.8566(51)$ \\
$\theta$ & radians & $1.963(11)$ & $-1.964(10)$ \\
$q$ & $10^{-3}$ & $0.950(33)$ & $0.952(33)$ \\
$t_{*}$ & days & $0.361(30)$ & $0.372(29)$ \\
$\pi_{E, N}$ & $\ldots$ & $-0.171(20)$ & $0.188(22)$ \\
$\pi_{E, E}$ & $\ldots$ & $-0.022(8)$ & $-0.040(10)$ \\
$\pi_{E}$ & $\ldots$ & $0.172(21)$ & $0.192(23)$ \\
$t_{0 s 2}$ & HJD -2455400 & $-0.08(60)$ & $0.22(9)$ \\
$u_{0 s 2}$ & $\ldots$ & $-0.267(19)$ & $0.301(19)$ \\
$f_{s 2 I}$ & $\ldots$ & $0.663(96)$ & $0.731(92)$ \\
$f_{s 2 V}$ & $\ldots$ & $0.801(117)$ & $0.877(113)$ \\
$d t_{E s 2}$ & days & $-1.2(11.6)$ & $-8.2(8.5)$ \\
$d \theta_{s 2}$ & radians & $0.305(35)$ & $-0.300(39)$ \\
$\omega$ & $10^{-3}$ days $^{-1}$ & $0.79(1.21)$ & $-1.17(1.17)$ \\
$\dot{s}$ & $10^{-3}$ days $^{-1}$ & $-1.76(30)$ & $-1.76(30)$ \\
$1 / T_{S \text { orb }}$ & $10^{-3}$ days $^{-1}$ & $0.50(13)$ & $0.49(12)$ \\
$\theta_{E}$ & mas & $0.805(100)$ & $0.777(95)$ \\
$\mu_{\text {rel,G }}$ & mas yr ${ }^{-1}$ & $2.46(31)$ & $2.44(31)$ \\
$V_{s 1}$ & $\ldots$ & $20.43(7)$ & $20.38(6)$ \\
$I_{s 1}$ & $\ldots$ & $17.95(7)$ & $17.90(6)$ \\
$K_{s 1}$ & $\ldots$ & $14.90(8)$ & $14.85(7)$ \\
$V_{s 2}$ & $\ldots$ & $20.69(11)$ & $20.53(10)$ \\
$I_{s 2}$ & $\ldots$ & $18.41(11)$ & $18.25(10)$ \\
$K_{s 2}$ & $\ldots$ & $15.58(14)$ & $15.42(14)$ \\
$K_{s 12}$ & $\ldots$ & $14.43(4)$ & $14.34(4)$ \\
\hline & & &
\end{tabular}

$t_{*}>0.30$ days. Also, large values of $\left|d t_{E s 2}\right|$ and $\left|d \theta_{s 2}\right|$ are excluded for the smallest $t_{*}$ values. The microlensing parallax amplitude, $\pi_{E}$, is not strongly correlated with any of the source or lens orbital motion parameters. It does have a strong anticorrelation with the Einstein radius crossing time, but this is just a well-known feature of the blending degeneracy that is responsible for the uncertainty in $t_{E}$.
The $\chi^{2}$ difference between the $u_{0}>0$ and $u_{0}<0$ solutions is small, as indicated in the bottom row of Table 1 . The $u_{0}>0$ solution is best, with the best $u_{0}<0$ solution disfavored by $\Delta \chi^{2}=4.21$. This small $\chi^{2}$ differences imply that all of these solutions will contribute to the physical parameter probability distributions, but the $u_{0}>0$ solutions will dominate.

An unusual feature of this event is that the source system consists of two stars that have both left the main sequence. In contrast to the situation for main-sequence stars, the fainter star is bluer than the brighter star for most of the solutions that comprise our Markov chains. This can be seen from Table 2 and even more clearly in the color-magnitude diagram (CMD) shown in Figure 7. This will allow us to constrain the source distance by requiring that the source stars lie on the same isochrone in Section 5.

\section{Photometric Calibration and Primary Source Radius}

In order to measure the angular Einstein radius, $\theta_{E}=$ $\theta_{*} t_{E} / t_{*}$, we must determine the angular radius of the source star, $\theta_{*}$, from the dereddened brightness and color of the source star (Kervella et al. 2004; Boyajian et al. 2014). We determine the calibrated source brightness in the $V$ and $I$ bands by calibrating the OGLE-IV light curve photometry to the OGLEIII catalog (Szymański et al. 2011). This gives

$$
\begin{gathered}
V_{\mathrm{O} 3 \mathrm{cal}}=0.2643+V_{\mathrm{O} 4}-0.0855\left(V_{\mathrm{O} 4}-I_{\mathrm{O} 4}\right), \\
I_{\mathrm{O} 3 \mathrm{cal}}=0.0403+I_{\mathrm{O} 4}+0.0032\left(V_{\mathrm{O} 4}-I_{\mathrm{O} 4}\right),
\end{gathered}
$$

where $V_{\mathrm{O} 4}$ and $I_{\mathrm{O} 4}$ are the OGLE-IV light curve magnitudes, and $V_{\mathrm{O} 3 \mathrm{cal}}$ and $I_{\mathrm{O} 3 \mathrm{cal}}$ are the calibrated OGLE-III magnitudes.

In order to estimate the source radius, we need extinctioncorrected magnitudes, and we determine these from the magnitudes and colors of the centroid of the red clump giant feature in the OGLE-III CMD, as indicated in Figure 7. Using the red clump centroid-finding method of Bennett et al. (2010), we find the red clump centroid to be located at $I_{\mathrm{O} 3 \mathrm{rc}}=15.868$ and $V_{\mathrm{O} 3 \mathrm{rc}}-I_{\mathrm{O} 3 \mathrm{rc}}=2.475$. We compare this to the predicted extinction-corrected red clump centroid magnitude and color of 


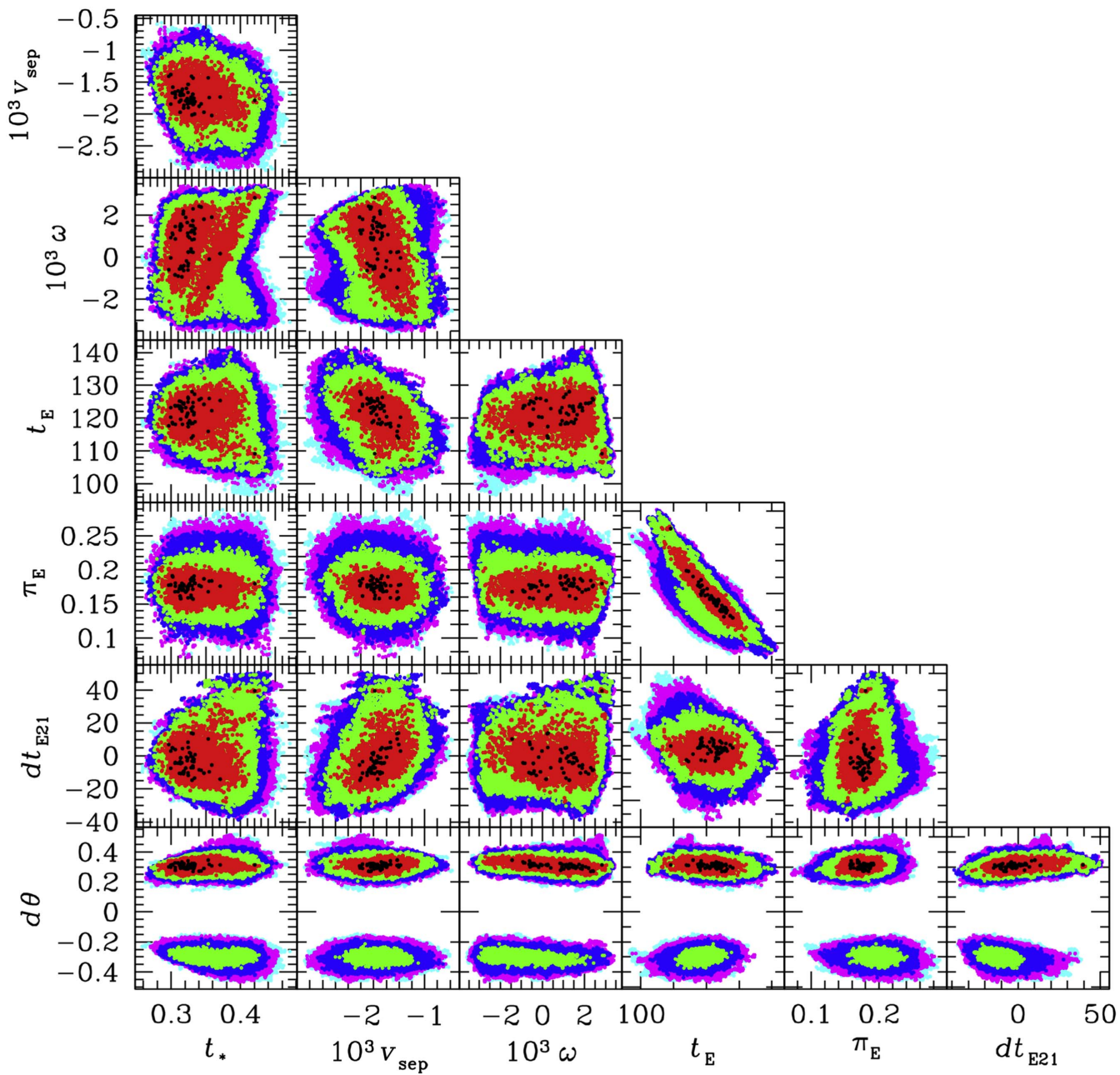

Figure 6. Correlations from our MCMC runs between lens velocity parameters ( $v_{\text {sep }}$ and $\omega$ ) and the parameters that affect the inferred host star mass: $d \theta, t_{*}, t_{E}, \pi_{E}$, and $d t_{E 21}$, from our MCMC runs. Smaller $t_{*}$ values imply larger $\theta_{E}$ values, which imply tighter constraints on the parameters that describe the source velocities, $d \theta$, and $d t_{E 21}$. Black, red, green, blue, magenta, and cyan indicate models that have $\chi^{2}$ values larger than the best-fit model by $\Delta \chi^{2}<1,1<\Delta \chi^{2}<4,4<\Delta \chi^{2}<9$, $9<\Delta \chi^{2}<16,16<\Delta \chi^{2}$, and $\Delta \chi^{2}>16$, respectively.

$I_{\mathrm{rc} 0}=14.288$ and $V_{\mathrm{rc} 0}-I_{\mathrm{rc} 0}=1.06$, which is appropriate (Bensby et al. 2013; Nataf et al. 2013) for the Galactic coordinates of this event, $(l, b)=(5.5875,-2.4680)$. This yields extinction values of $A_{I}=1.580$ and $A_{V}=2.995$, which implies an extinction-corrected primary source magnitude and colors of $I_{s 1,0}=16.421$ and $V_{s 1,0}-I_{s 1,0}=1.052$ for the best-fit model.

These dereddened magnitudes can be used to determine the angular source radius, $\theta_{*}$. We use the relation from the analysis of Boyajian et al. (2014), but with a restricted range of colors corresponding to $3900<T_{\text {eff }}<7000 \mathrm{~K}$ (T. Boyajian 2014, private communication). We use

$$
\begin{aligned}
& \log _{10}\left[2 \theta_{*} /(1 \mathrm{mas})\right] \\
& \quad=0.501414+0.419685(V-I)_{s 1,0}-0.2 I_{s 1,0},
\end{aligned}
$$

and this gives $\theta_{*}=2.20 \mu$ as for the best-fit model. Now, there is some indication of differential reddening in the CMD (Figure 7), so this can add some uncertainty to our determination of $\theta_{*}$. Fortunately, the effect of this uncertainty in the extinction tends to cancel contributions from $(V-I)_{s 1,0}$ and $I_{s 1,0}$ in Equation (5). To account for this uncertainty, we add a $13 \%$ uncertainty to our extinction estimates, which translates into a $9 \%$ uncertainty in $\theta_{*}$, according to
Equation (5), to be used in our MCMC calculations. As Figure 7 indicates, the uncertainty in the magnitude and color of source 1 is larger than the uncertainty for most events. This is because flux can be traded between the two sources. However, this source radius determination is correlated with the other microlens model parameters, particularly the Einstein radius crossing time, $t_{E}$, which occurs in the $\theta_{E}=\theta_{*} t_{E} / t_{*}$ formula. Therefore, we determine $\theta_{E}$ for each model in our MCMC, and this yields the $\theta_{E}$ values listed in Table 2: $\theta_{E}=0.805 \pm 0.100 \mathrm{mas}$ for the $u_{0}>0$ solutions and $\theta_{E}=0.777 \pm 0.095$ mas for the $u_{0}<0$ solutions.

\section{Lens System Properties}

When both the angular Einstein radius, $\theta_{E}$, and the microlensing parallax, $\pi_{E}$, are measured, we can use the following relation (Gould 1992; An et al. 2002; Gould et al. 2004),

$$
M_{L}=\frac{\theta_{E} c^{2} \mathrm{au}}{4 G \pi_{E}}=\frac{\theta_{E}}{(8.1439 \mathrm{mas}) \pi_{E}} M_{\odot},
$$

to determine the mass of the lens system, but in our case, we have degenerate solutions to consider. The degeneracy 


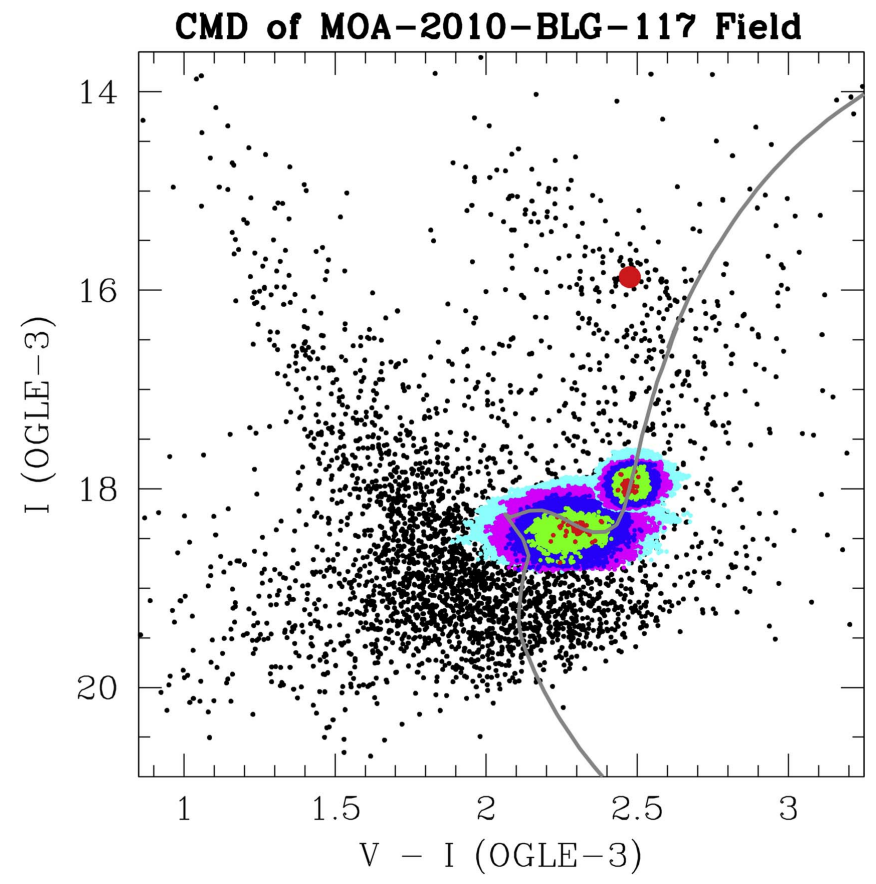

Figure 7. $(V-I, I)$ color-magnitude diagram (CMD) of the stars in the OGLE-III catalog (Szymański et al. 2011) within 90" of MOA-2010-BLG-117. The red spot indicates the red clump giant centroid, and the smaller spots of different colors indicate the magnitude and colors of the two sources from our MCMC calculations. Red, green, blue, magenta, and cyan indicate models that have $\chi^{2}$ values larger than the best-fit model by $\Delta \chi^{2}<1,1<\Delta \chi^{2}<4$, $4<\Delta \chi^{2}<9,9<\Delta \chi^{2}<16$, and $16<\Delta \chi^{2}$, respectively. Source 1 is brighter and redder than source 2 for most models. The gray line indicates the isochrone that best matches the source magnitudes and colors of the best-fit model. This isochrone has an age of $4.0 \mathrm{Gyr}$ and a metallicity of $[\mathrm{Fe} / \mathrm{H}]=0.28$.

allowing different $t_{*}$ values is probably unique to the specific circumstances of this event. However, the degeneracy between the $u_{0}>0$ and $u_{0}<0$ solutions is a very common degeneracy due to the reflection of the lens plane with respect to the orientation of the Earth's orbit, which allows us to measure the parallax effect with ground-based data. For high-magnification events, the lens-source system has an approximate reflection symmetry, so this $u_{0}>0 \leftrightarrow u_{0}<0$ degeneracy has little effect on $\pi_{E} \equiv\left|\pi_{E}\right|$. Because the binary source system for MOA-2010-BLG-117 has $u_{0} \approx-u_{0 s 2}$ and source 2 is only $\sim 0.3 \mathrm{mag}$ fainter than source 1 , the lens-and-source system in this event also has an approximate symmetry (assuming that the planetary feature has little influence on the microlensing parallax signal). This could be the reason why the distributions of the $\pi_{E}$ vector, shown in Figure 8, also show this approximate reflection symmetry. This figure shows the distributions from both degenerate solutions with best-fit parameters listed in Table 1 and Markov chain distributions listed in Table 2. The $u_{0}>0$ and $u_{0}<0$ solutions are widely separated with opposite signs for the $\pi_{E, N}$ values. These opposite signs mean that the $\left|\pi_{E, N}\right|$ values are very similar for all solutions. The $\pi_{E, E}$ values are also similar and much smaller than $\left|\pi_{E, N}\right|$, so the $\pi_{E}$ values for all the degenerate solutions are similar. This means that there is overlap in the mass distributions predicted by all four degenerate solutions.

As mentioned in Section 3, we impose a requirement that both sources lie on the same isochrone. This requirement is not

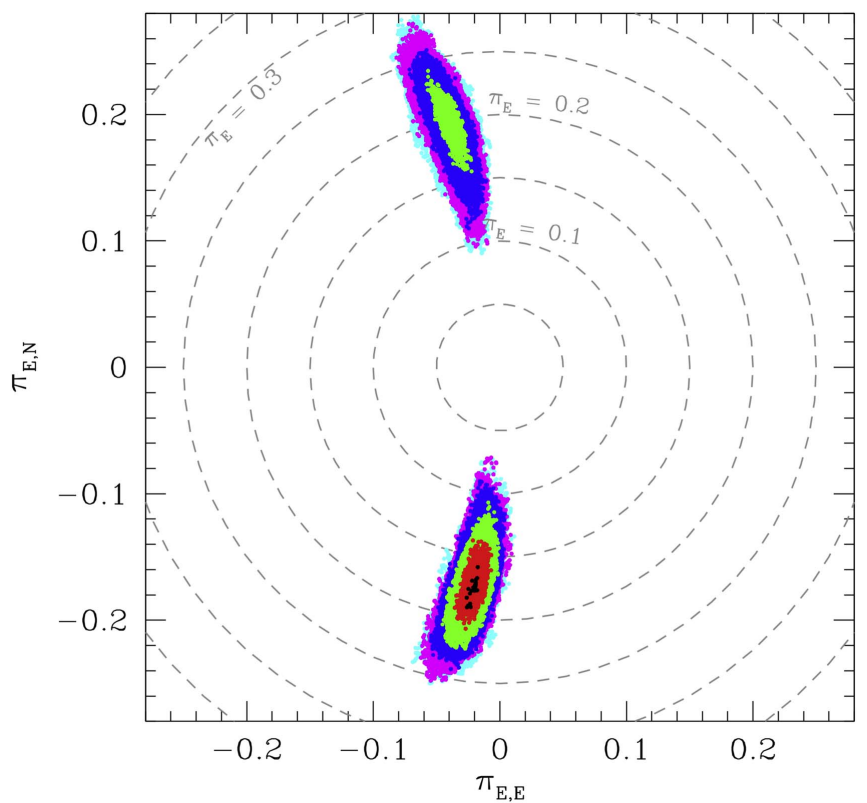

Figure 8. Values of the microlensing parallax vector, $\pi_{E}$, from our MCMC runs are shown. The $u_{0}>0$ solutions have $\pi_{E, N}<0$ and are preferred over the $u_{0}<0$ solutions (with $\pi_{E, N}>0$ ) by $\Delta \chi^{2}=9.17$. The MCMC points are colorcoded. The points within $\Delta \chi^{2}<1$ are black, and the points within $1<\Delta \chi^{2}<4, \quad 4<\Delta \chi^{2}<9, \quad 9<\Delta \chi^{2}<16, \quad 16<\Delta \chi^{2}<25$, and $25<\Delta \chi^{2}$ are red, green, blue, magenta, and cyan, respectively. The dashed circles indicate curves of constant $\pi_{\mathrm{E}}$.

imposed during the light curve modeling, but it is imposed in our Bayesian analysis that uses all of the models from our Markov chains to determine the physical parameters of the lens system. Each light curve model in our Markov chains is weighted by the $\chi^{2}$ of the best fit of the model source magnitudes and colors to the isochrones. Thus, the location of the source magnitudes and colors in Figure 7 does not depend on these isochrones, but the color-coding of the source magnitudes and colors does depend on the depend on the fit to the isochrones. We use isochrones from the PAdova and TRieste Stellar Evolution Code (PARSEC) project (Bressan et al. 2012; Chen et al. 2014, 2015; Tang et al. 2014). We find that our modeling results are consistent with isochrones with ages in the range of $4-10 \mathrm{Gyr}$ and metallicity in the range of $-0.04 \leqslant[\mathrm{Fe} / \mathrm{H}] \leqslant 0.58$. These values are quite typical of Galactic bulge stars, as indicated by the microlens source stars with high-resolution spectra taken at high magnification by Bensby et al. (2013, 2017). However, it is also possible that the source might have a slightly higher extinction than the average of the red clump stars. In that case, the range of allowed source star metallicities might extend to subsolar metallicities.

The main practical effect of this isochrone constraint is to force the source star system to be located on the near side of the bulge. The isochrones prefer a source distance of $D_{S}=$ $6.2 \pm 1.1 \mathrm{kpc}$, but when these priors on the source density and microlensing probability are included, this shifts to $D_{S}=6.9 \pm 0.7 \mathrm{kpc}$, as given in Table 3 .

We determine the physical parameters of this lens system with a Bayesian analysis marginalized over the Galactic model used by Bennett et al. (2014), and the results are summarized in Figures 9 and 10, as well as in Table 3. The host star and planet masses $\left(M_{h}\right.$ and $\left.m_{p}\right)$ are determined directly from Equation (6) with the $\pi_{E}, q$, source magnitude, and color values determined for each model in our MCMC. The $\theta_{*}$ and $\theta_{E}$ values are determined 
Table 3

Physical Parameters

\begin{tabular}{llcc}
\hline \hline Parameter & Units & Value & $2-\sigma$ range \\
\hline$D_{S}$ & $\mathrm{kpc}$ & $6.9 \pm 0.7$ & $5.6-8.3$ \\
$D_{L}$ & $\mathrm{kpc}$ & $3.5 \pm 0.4$ & $2.9-4.3$ \\
$M_{h}$ & $M_{\odot}$ & $0.58 \pm 0.11$ & $0.39-0.83$ \\
$m_{p}$ & $M_{\text {Jup }}$ & $0.54 \pm 0.10$ & $0.38-0.77$ \\
$a_{\perp}$ & $\mathrm{au}$ & $2.42 \pm 0.26$ & $1.93-2.97$ \\
$a_{3 d}$ & $\mathrm{au}$ & $2.9_{-0.6}^{+1.6}$ & $2.1-10.3$ \\
$V_{L}$ & $\mathrm{mag}$ & $24.3_{-1.7}^{+1.5}$ & $21.1-27.2$ \\
$I_{L}$ & $\mathrm{mag}$ & $21.2_{-1.1}^{+1.0}$ & $19.1-23.0$ \\
$K_{L}$ & $\mathrm{mag}$ & $18.3_{-0.8}^{+0.6}$ & $16.7-19.6$ \\
\hline
\end{tabular}

Note. Uncertainties are $1 \sigma$ parameter ranges.

directly from Equations (5), (3), and (4) for each model. The $u_{0}<0$ solutions are weighted by $e^{-\Delta \chi^{2} / 2}=0.122$ with respect to the $u_{0}>0$ solutions, where $\Delta \chi^{2}=4.21$ is the $\chi^{2}$ difference between the best-fit solutions with the parameters listed in Table 1. There is no appreciable difference in the parameter space volume covered by the two solutions, so this approach is adequate. The Galactic model prior has little influence on the lens mass determination because the prior has little variation over the parameter values that are consistent with the MCMC light curve models. The Galactic model has a larger influence on the distance to the lens, because the stellar density has a strong dependence on the distance to the source star, $D_{S}$. The relation between the distances to the lens and source stars is given by

$$
D_{L}=\frac{\mathrm{au}}{\pi_{E} \theta_{E}+\pi_{S}}
$$

where $\pi_{S}$ is the parallax of the source star, $\pi_{S}=\mathrm{au} / D_{S}$. As Table 3 indicates, these calculations indicate that the host star has a mass of $M_{h}=0.58 \pm 0.10 M_{\odot}$ and the planet has a mass of $M_{p}=0.51 \pm 0.07 M_{\mathrm{Jup}}$, where $M_{\mathrm{Jup}}$ is the mass of Jupiter. Assuming a random orientation, their three-dimensional separation is $a_{3 d}=2.9_{-0.5}^{+1.6}$ au. The planet mass uncertainty is smaller than the host mass uncertainty because the high host star mass $\left(t_{*}<0.34\right.$ days $), u_{0}>0$ solutions have a lower mass ratio than the other solutions, as indicated in Tables 1 and 2.

The predicted host (and lens) star $V, I$, and $K$ magnitudes are shown in Figure 10, along with the source distance, $D_{S}$. The distribution of $D_{S}$ favors a large number of discrete values. This is due to our requirement that the two source stars lie on the same isochrone and the discrete values of the metallicity, $[\mathrm{Fe} / \mathrm{H}]$, and $\log ($ Age $)$ at intervals of 0.04 and 0.05 , respectively.

The additional source star also increases our odds of detecting planets orbiting the lens star because the second source provides a second probe of the lens plane. This can be seen in Figure 11, which shows the two cases from our recent exoplanet mass ratio function paper (Suzuki et al. 2016). Over much of the parameter range, the second source star approximately doubles the planet detection efficiency. However, this is a much smaller increase than is provided by highmagnification events.

\section{Keck Follow-up Observations}

In an attempt to identify the lens and planetary host star, we have obtained high angular resolution AO observations from the Keck II telescope. Unfortunately, the seeing conditions
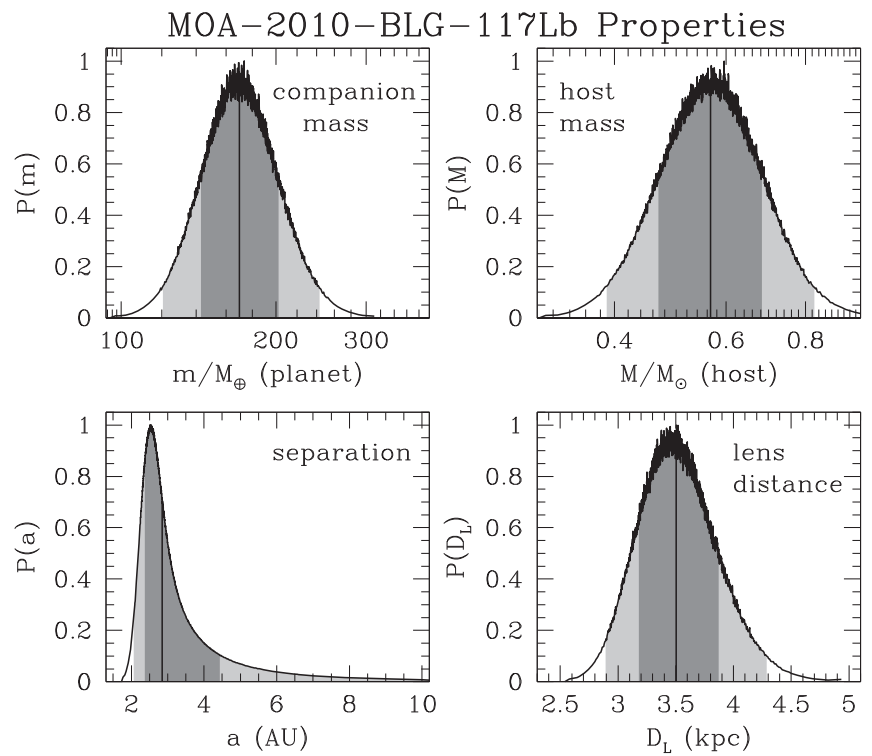

Figure 9. Probability distributions of the planet and host star mass, threedimensional separation and lens system distance based on a Bayesian analysis using mass and distance determinations from the MCMC light curve distributions along with prior probabilities from a standard Galactic model.
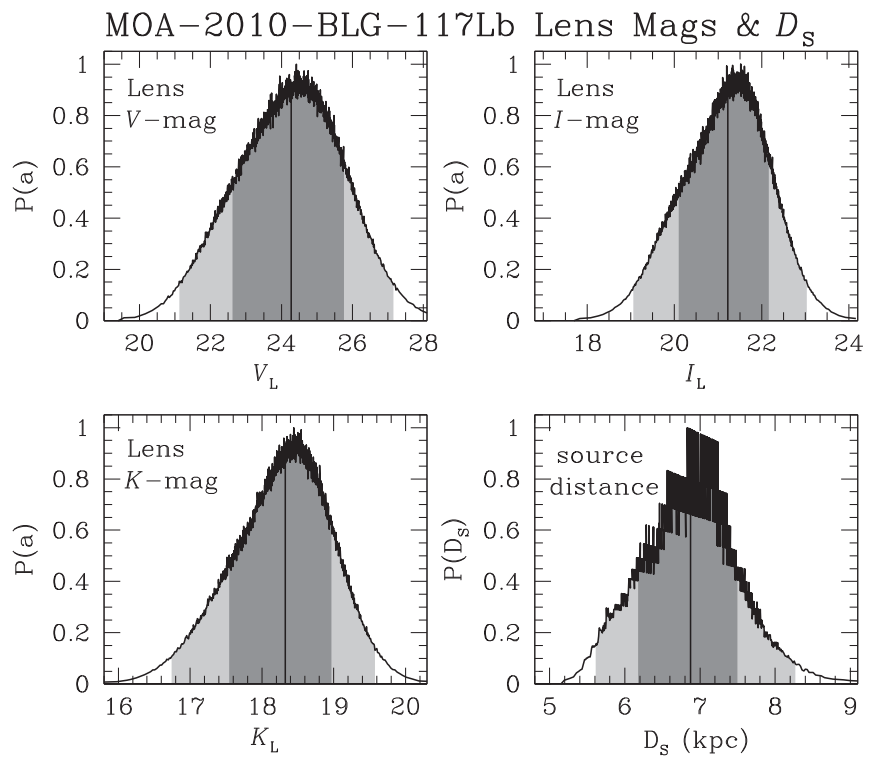

Figure 10. Probability distributions of the host star $V, I$, and $K$ magnitudes, based on our light curve models and Bayesian priors from a standard Galactic model.

were relatively poor compared to some of our other Keck observations (Batista et al. 2015) that achieved a point-spread function (PSF) FWHM of 60 mas. Our stacked $K$-band image of the MOA-2010-BLG-117 field has a PSF FWHM of 220 mas, and it is shown in Figure 12. The Keck images were taken in 2012, two years after the event. With a lens-source relative proper motion of $\mu_{\text {rel, G }}=2.45 \pm 0.31 \mathrm{mas} \mathrm{yr}^{-1}$, there is no chance to detect the lens-source separation either through image elongation (Bennett et al. 2007, 2015) or a colordependent image centroid shift (Bennett et al. 2006). However, there is still a chance to detect the unresolved lens star flux on top of the flux from the source stars. In this case, the source stars are relatively bright subgiants, so it would be difficult to detect a host star as faint as the star indicated by the finite 

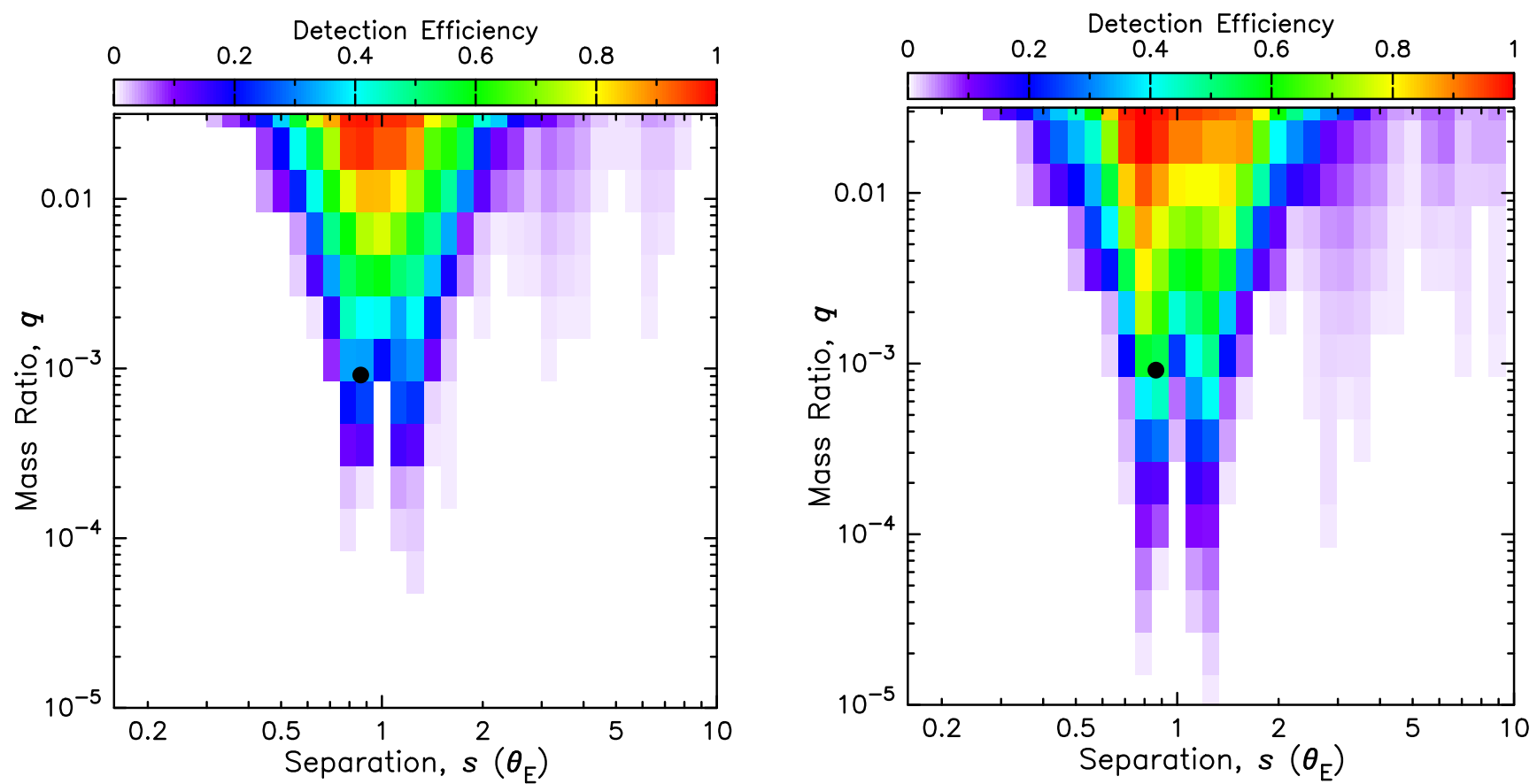

Figure 11. Planetary detection efficiency for MOA-2010-BLG-117. The left panel shows the detection efficiency due to the source star that led to the real planet detection, and the right panel shows the planet detection efficiency for the actual event with both source stars. In both cases, the black spots indicate the position of the planet.

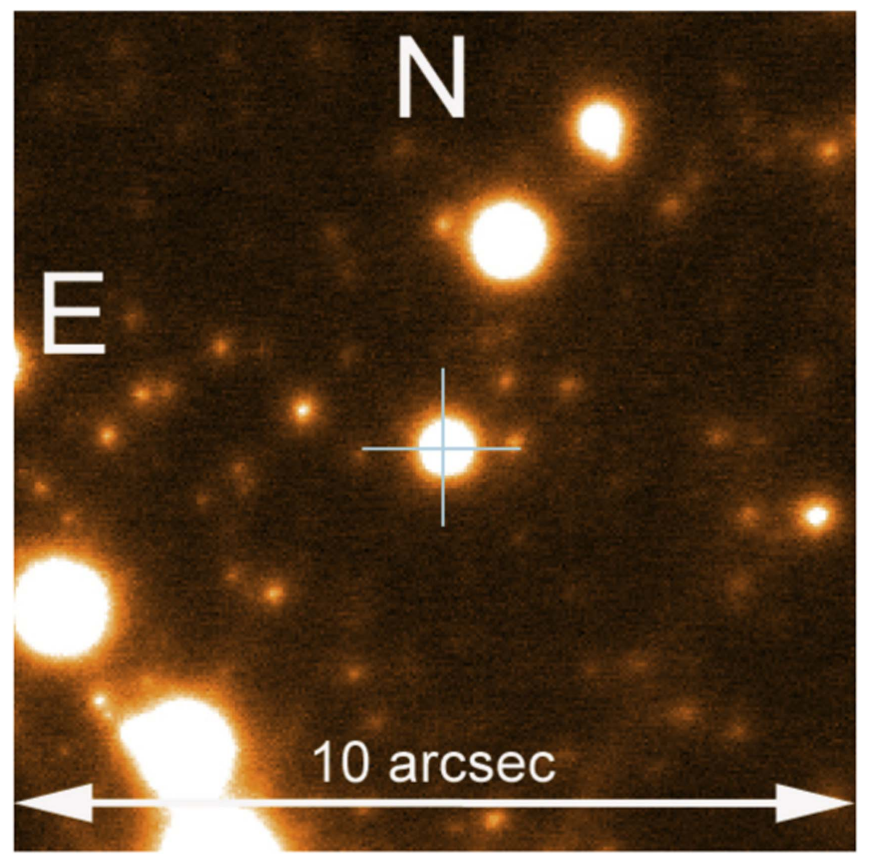

Figure 12. Co-added Keck AO image of the target star indicated by the crosshairs. The target consists of the combined flux of the source stars, the lens (and planetary host star), any bound companions to either the source or lens system, and possibly an unrelated star that happens to be located $\lesssim 0$ ". 2 from the source.

source and microlensing parallax measurements, as discussed in Section 5.

The "star" detected in the Keck AO images is indeed significantly brighter, $K_{\text {Keck }}=13.97 \pm 0.04$, than the combined flux of the source stars, which is $K_{s 12}=14.43 \pm 0.04$. However, this excess blend flux at $K_{b}=15.12 \pm 0.15$ does not match the lens mass and distance derived in Section 5. The

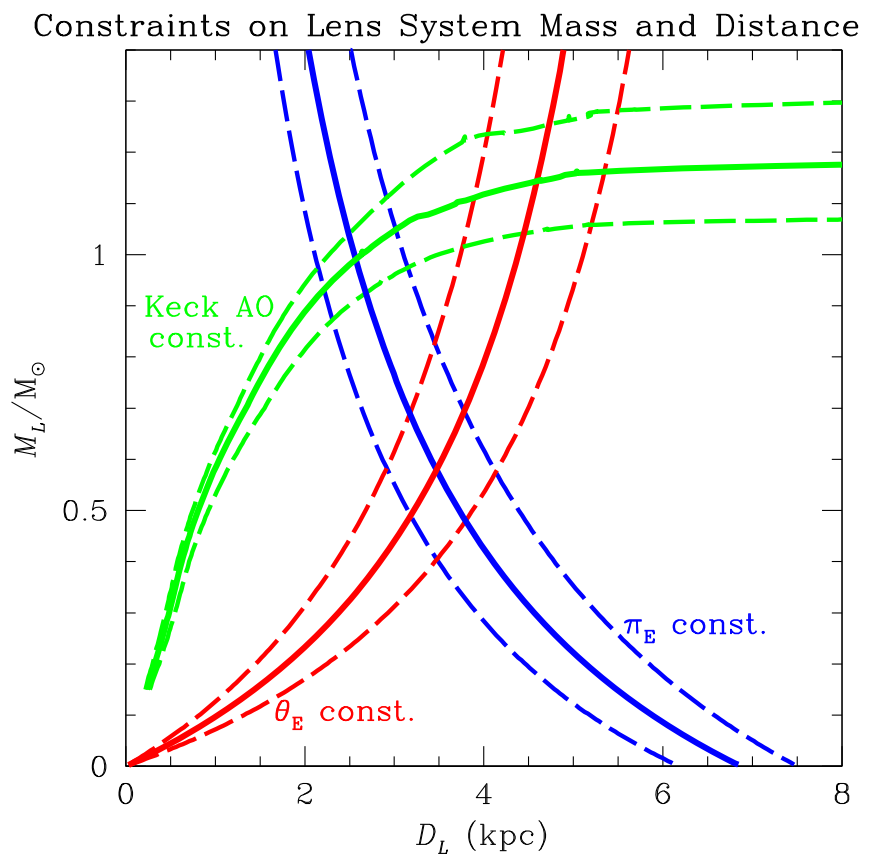

Figure 13. Constraints on the host star mass and distance from the microlensing parallax, $\pi_{E}$ (in blue), the angular Einstein radius, $\theta_{E}$ (in red), and the host star flux (in green), under the assumption that the excess flux observed in the Keck AO images is due to the host star. The dashed line indicates the approximate $1 \sigma$ uncertainty contours (which ignore the correlations between the parameters). For the excess $K$-band flux, the solid green line is from a $5 \mathrm{Gyr}$ isochrone and the dashed green lines represent 3 and 7 Gyr isochrones (Bressan et al. 2012; Chen et al. 2014, 2015; Tang et al. 2014).

predicted host star brightness is $K_{L}=18.3_{-0.8}^{+0.6}$, and as can be seen from Figure 10, the probability of the lens (and host) star being brighter than $K_{L}<16$ is negligible. Figure 13 compares the constraints from the microlensing parallax, angular Einstein 
radius, and lens flux constraints, assuming that the excess flux is due to the lens star. Obviously, these constraints are not consistent with each other. The most likely solution to this inconsistency is simply that the excess flux is not due to the lens. The other possibilities that could explain this excess flux at the position of the source star are a binary companion to the lens, a tertiary companion to the source stars, or an unrelated star. A Bayesian analysis using the measured bulge luminosity function and measured frequencies of multiple star systems (N. Koshimoto et al. 2017, in preparation) gives similar probabilities for each of these possibilities, with slightly larger probabilities for lens and source companions than for an unrelated star.

Although we believe that the result from the $\pi_{E}$ and $\theta_{E}$ measurements is very likely to be the correct interpretation, we will briefly consider that one of these measurements is wrong. From Figure 13, we see that a host star mass of $M_{h} \sim 1 M_{\odot}$ at a distance of $D_{L} \sim 2.6 \mathrm{kpc}$ would be favored if the blend flux is due to the lens star and the $\pi_{E}$ measurement is correct. Alternatively, if the $\pi_{E}$ measurement were incorrect, while the $\theta_{E}$ measurement were correct and the blend flux were due to the lens star, then the lens star would have to be an evolved star above a solar mass. The green isochrone curves in Figure 13 are nearly horizontal where they cross the red $\theta_{E}=$ const. curve. This is due to the fact that stars evolve very quickly through these evolved phases, and this implies that this solution is particularly unlikely.

A final possibility is that the $\pi_{E}$ and $\theta_{E}$ measurements are correct, and that the excess flux comes from the planetary host star. This would imply that the assumption made for the red and blue $\theta_{E}$ and $\pi_{E}$ curves in Figure 13 that the source is in the Galactic bulge (at $D_{S}=6.8 \pm 0.6 \mathrm{kpc}$ ) is not correct. From Equation (7), we have $D_{S}=D_{L} /\left(1-\pi_{E} \theta_{E} D_{L} / \mathrm{au}\right)$, and this tells us that if the lens system is located at $D_{L} \approx 0.9$ where the green lens flux curve crosses the $M_{h}=0.58 \pm 0.10$ value indicated by the $\theta_{E}$ and $\pi_{E}$ measurements (according to Equation (6)), then the source would be at a distance of $D_{S}=1.04 \mathrm{kpc}$. This is highly unlikely or at least ruled out for two reasons. First, the rate that stars at this distance are microlensed is more than two orders of magnitude lower than the rate that bulge stars are microlensed. Second, the two source stars appear to reside on the Galactic bulge subgiant branch of the CMD, shown in Figure 7. Very few foreground disk stars lie on this portion of the CMD, and there is virtually no way to arrange for the fainter star in a binary pair to be bluer than the brighter star.

Another indication that the source stars must reside in the Galactic bulge comes from the proper motion of the source star system. Skowron et al. (2014) has developed a method to determine the proper motion of microlens source stars in the presence of a modest amount of blending with other stars. We have used this method to measure the proper motions of stars brighter than $I<17.87$, for just over five years of OGLE-IV data. (This magnitude cut is two magnitudes below the red clump centroid.) Figure 14 shows that the proper motion of the target, consisting of the two source stars and a blend star with a magnitude of about the average of the two source stars. If the blend star were the lens, it would be in the Galactic disk, so we would expect the average proper motion of the two source stars and the blend to be shifted slightly in the direction of the disk rotation (given by the dashed white line in the NNE direction). Instead, we find the proper motion of the target to be

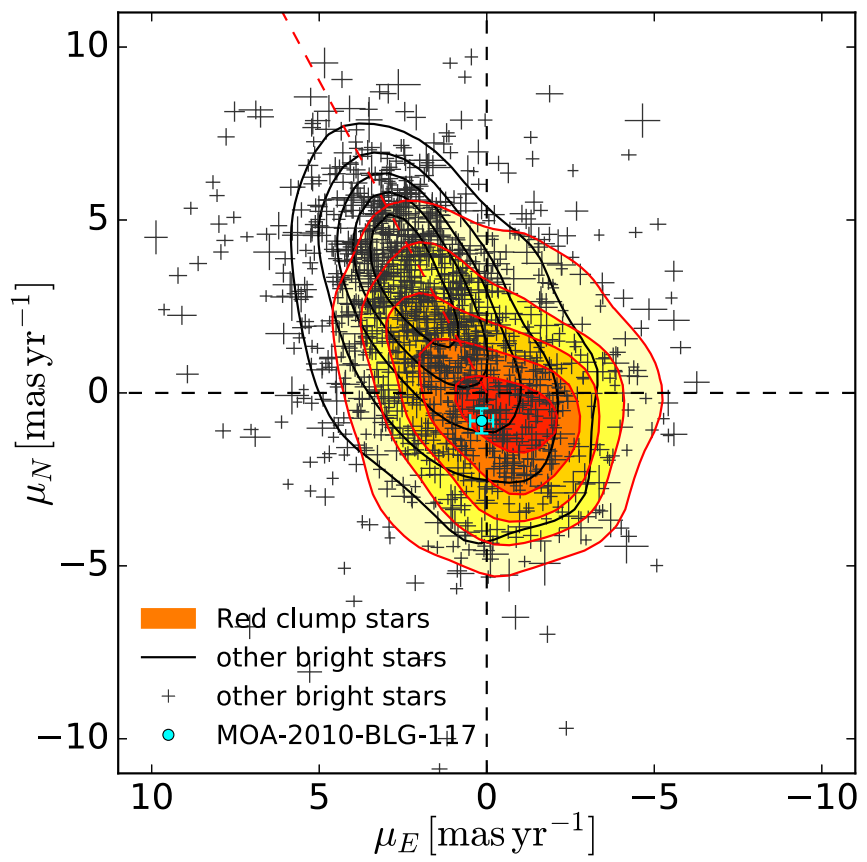

Figure 14. Proper motions of stars brighter than the source stars plus the blend star from the OGLE-IV survey. The red and yellow shaded contours indicate the proper motion distribution of 922 bulge red clump stars, and the black contours indicate the distribution of the remaining 2239 stars brighter than $I<17.87$. The red clump stars come from a compact elliptical region of the CMD, elongated in the direction of the extinction vector. The dashed red line extending in the NNE direction indicates the direction of Galactic rotation, so we expect the distribution of Galactic disk stars to be extended in the direction of this line. The cyan-colored spot with error bars in both the $\mathrm{E}$ and $\mathrm{N}$ directions indicates the target. The target consists of the two bound source stars and a blend star similar in brightness to each of the two source stars. The proper motion of the source stars indicates that they are likely to be bulge stars.

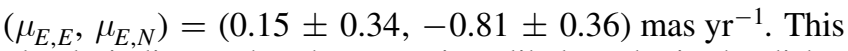
clearly indicates that the target is unlikely to be in the disk. Of course, it could be that the blend star and the two source stars are not in the same population, and their proper motions could partially cancel. However, our light curve modeling indicates that the lens-source relative proper motion is in the range of 2-3 mas $\mathrm{yr}^{-1}$, so if the blend star were the lens, its proper

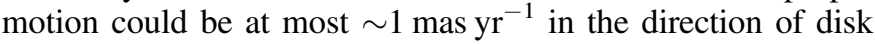
rotation. Thus, it would not have disk kinematics. This tends to confirm our conclusion that the blend star cannot be the lens.

So, we conclude that the source star system resides in the Galactic bulge and that the host star mass and the lens system distance are determined by the $\pi_{E}$ and $\theta_{E}$ measurements, as described in Section 5.

\section{Discussion and Conclusions}

We have presented the first planetary microlensing event with two magnified source stars. This event has an obvious planetary feature, but it could not be modeled with a single source star microlensed by a lens system consisting of one star and one planet. The basic properties of the planetary feature could be explained by models with two source stars or else a circumbinary planet. The choice between these two options was delayed by early difficulties in modeling the event. These difficulties were overcome by adding the requirement that the flux ratio between the two sources be consistent with different passbands to allow the best light curve model to be found much more easily. The finite source effects and microlensing parallax 
signal indicate that the planet and host have masses of $m_{p}=0.58 \pm 0.10 M_{\text {Jup }}$ and $M_{h}=0.51 \pm 0.07 M_{\odot}$ at a twodimensional separation of $a_{\perp}=2.44 \pm 0.26$ au and a distance of $D_{L}=3.4 \pm 0.2 \mathrm{kpc}$. This is a Jupiter-mass-ratio planet orbiting at about twice the distance of the snow line, which is similar to Jupiter's orbit.

One complication in the interpretation of this event is the $K$-band Keck AO images that indicate an excess of flux at the location of the source. This excess flux is much brighter than the brightness expected from the lens star, based on the mass determined from the $\theta_{E}$ and $\pi_{E}$ measurements. We consider the possibility that this excess flux could be due to the lens, but we find that the excess flux is more likely to be due to a companion to the lens star, the source stars, or an unrelated star. This is not the first planetary microlensing event with a binary source star, as the planetary event OGLE-2007-BLG-368 (Sumi et al. 2010) has a binary source star that was revealed via the xallarap effect. (Xallarap is the effect of source orbital motion on the microlensing light curve.)

This event was as challenging to model as events with an additional lens mass, either a second star (Gould et al. 2014; Poleski et al. 2014; Bennett et al. 2016) or a second planet (Gaudi et al. 2008; Bennett et al. 2010; Han et al. 2013; Beaulieu et al. 2016). However, events with an additional lens mass have interesting implications regarding the properties of exoplanet systems, while events with two source stars do not. The only advantage of a second source star is a modest increase in the exoplanet detection efficiency. Nevertheless, microlensing is currently our best method for understanding the population of exoplanets that orbit beyond the snow line, and the statistical analysis of the planet populations probed by the microlensing method requires that the correct microlensing model be found for all planetary microlensing events. The new method that we have presented in this paper aids in this effort, and it has enabled the MOA Collaboration analysis that has discovered a break in the exoplanet mass ratio function (Suzuki et al. 2016).

D.P.B., A.B., and D.S. were supported by NASA through grant NASA-NNX12AF54G. This work was partially supported by a NASA Keck PI Data Award, administered by the NASA Exoplanet Science Institute. Data presented herein were obtained at the W. M. Keck Observatory from telescope time allocated to the National Aeronautics and Space Administration through the agency's scientific partnership with the California Institute of Technology and the University of California. The Observatory was made possible by the generous financial support of the W. M. Keck Foundation. The OGLE Team thanks Profs. M. Kubiak and G. Pietrzyński for their contribution to the collection of OGLE photometric data. The OGLE project has received funding from the National Science Centre, Poland, grant MAESTRO 2014/14/A/ST9/00121 to A.U. The work by C.R. was supported by an appointment to the NASA Postdoctoral Program at the Goddard Space Flight Center, administered by USRA through a contract with NASA. The work by N.K. is supported by JSPS KAKENHI Grant Number JP15J01676. A.G. and B.S.G. were supported by NSF grant AST 110347 and by NASA grant NNX12AB99G.

\section{ORCID iDs}

D. P. Bennett (1) https://orcid.org/0000-0001-8043-8413

C. Han (1) https://orcid.org/0000-0002-2641-9964
J.-P. Beaulieu (1) https://orcid.org/0000-0003-0014-3354

J. Skowron (1) https://orcid.org/0000-0002-2335-1730

B. S. Gaudi (1) https://orcid.org/0000-0003-0395-9869

N. Koshimoto (1) https://orcid.org/0000-0003-2302-9562

A. Fukui (1) https://orcid.org/0000-0002-4909-5763

Y. Itow (1) https://orcid.org/0000-0002-8198-1968

N. J. Rattenbury (ㄷ) https://orcid.org/0000-0001-5069-319X

D. Suzuki (1) https://orcid.org/0000-0002-5843-9433

R. W. Pogge (1) https://orcid.org/0000-0003-1435-3053

J. C. Yee (1) https://orcid.org/0000-0001-9481-7123

M. D. Albrow (i) https://orcid.org/0000-0003-3316-4012

E. Bachelet (1) https://orcid.org/0000-0002-6578-5078

V. Batista (i) https://orcid.org/0000-0002-9782-0333

J. A. R. Caldwell (i) https://orcid.org/0000-0002-3861-5241

K. Horne (1) https://orcid.org/0000-0003-1728-0304

M. Hundertmark @ https://orcid.org/0000-0003-0961-5231

N. Kains (1) https://orcid.org/0000-0001-8803-6769

S. R. Kane (i) https://orcid.org/0000-0002-7084-0529

K. C. Sahu (i) https://orcid.org/0000-0001-6008-1955

J. Wambsganss (1) https://orcid.org/0000-0002-8365-7619

\section{References}

Albrow, M. D., et al. 2009, MNRAS, 397, 2099

Alcock, C., Allsman, R. A., Alves, D., et al. 1995, ApJL, 454, L125 An, J. H., Albrow, M. D., Beaulieu, J.-P., et al. 2002, ApJ, 572, 521 Batista, V., Beaulieu, J.-P., Bennett, D. P., et al. 2015, ApJ, 808, 170 Beaulieu, J.-P., Bennett, D. P., Batista, V., et al. 2016, ApJ, 824, 83 Bennett, D. P. 2008, in Exoplanets, ed. J. Mason (Berlin: Springer) (arXiv:0902.1761)

Bennett, D. P. 2010, ApJ, 716, 1408

Bennett, D. P., Anderson, J., Bond, I. A., Udalski, A., \& Gould, A. 2006, ApJL, 647, L171

Bennett, D. P., Anderson, J., \& Gaudi, B. S. 2007, ApJ, 660, 781

Bennett, D. P., Batista, V., Bond, I. A., et al. 2014, ApJ, 785, 155

Bennett, D. P., Bhattacharya, A., Anderson, J., et al. 2015, ApJ, 808, 169

Bennett, D. P., Bond, I. A., Udalski, A., et al. 2008, ApJ, 684, 663

Bennett, D. P., \& Rhie, S. H. 1996, ApJ, 472, 660

Bennett, D. P., \& Rhie, S. H. 2002, ApJ, 574, 985

Bennett, D. P., Rhie, S. H., Nikolaev, S., et al. 2010, ApJ, 713, 837

Bennett, D. P., Rhie, S. H., Udalski, A., et al. 2016, AJ, 152, 125

Bennett, D. P., Sumi, T., Bond, I. A., et al. 2012, ApJ, 757, 119

Bensby, T., Feltzing, S., Gould, A., et al. 2017, arXiv:1702.02971

Bensby, T., Yee, J. C., Feltzing, S., et al. 2013, A\&A, 549, A147

Bertelli, G., Girardi, L., Marigo, P., \& Nasi, E. 2008, A\&A, 484, 815

Bond, I. A., Abe, F., Dodd, R. J., et al. 2001, MNRAS, 327, 868

Borucki, W. J., Koch, D. G., Basri, G., et al. 2011, ApJ, 736, 19

Boyajian, T. S., van Belle, G., \& von Braun, K. 2014, AJ, 147, 47

Bressan, A., Marigo, P., Girardi, L., et al. 2012, MNRAS, 427, 127

Burke, C. J., Christiansen, J. L., Mullally, F., et al. 2015, ApJ, 809, 8

Cassan, A., Kubas, D., Beaulieu, J.-P., et al. 2012, Natur, 481, 167

Chen, Y., Bressan, A., Girardi, L., et al. 2015, MNRAS, 452, 1068

Chen, Y., Girardi, L., Bressan, A., et al. 2014, MNRAS, 444, 2525

Gaudi, B. S. 2012, ARA\&A, 50, 411

Gaudi, B. S., Bennett, D. P., Udalski, A., et al. 2008, Sci, 319, 927

Gould, A. 1992, ApJ, 392, 442

Gould, A. 2008, ApJ, 681, 1593

Gould, A., Bennett, D. P., \& Alves, D. R. 2004, ApJ, 614, 404

Gould, A., Dong, S., Bennett, D. P., et al. 2010a, ApJ, 710, 1800

Gould, A., Dong, S., Gaudi, B. S., et al. 2010b, ApJ, 720, 1073

Gould, A., Gaudi, B. S., \& Han, C. 2004, arXiv:astro-ph/0405217

Gould, A., \& Loeb, A. 1992, ApJ, 396, 104

Gould, A., Udalski, A., Shin, I.-G., et al. 2014, Sci, 345, 46

Han, C., Bennett, D. P., Udalski, A., et al. 2016, ApJ, 825, 8

Han, C., Udalski, A., Choi, J.-Y., et al. 2013, ApJL, 762, L28

Han, C., Udalski, A., Gould, A., et al. 2017, AJ, 154, 223

Hwang, K.-H., Choi, J.-Y., Bond, I. A., et al. 2013, ApJ, 778, 55

Ida, S., \& Lin, D. N. C. 2005, ApJ, 626, 1045

Jung, Y. K., Udalski, A., Yee, J. C., et al. 2017, AJ, 153, 129

Kennedy, G. M., \& Kenyon, S. J. 2008, ApJ, 673, 502

Kennedy, G. M., Kenyon, S. J., \& Bromley, B. C. 2006, ApJL, 650, L139 
Kervella, P., Thévenin, F., Di Folco, E., \& Ségransan, D. 2004, A\&A, 426, 297

Lecar, M., Podolak, M., Sasselov, D., \& Chiang, E. 2006, ApJ, 640, 1115

Lissauer, J. J. 1993, ARA\&A, 31, 129

Mao, S., \& Paczyński, B. 1991, ApJL, 374, L37

Minniti, D., Lucas, P. W., Emerson, J. P., et al. 2010, NewA, 15, 433

Mullally, F., Coughlin, J. L., Thompson, S. E., et al. 2016, arXiv:1602.03204

Muraki, Y., Han, C., Bennett, D. P., et al. 2011, ApJ, 741, 22

Nataf, D. M., Gould, A., Fouqué, P., et al. 2013, ApJ, 769, 88

Pejcha, O., \& Heyrovský, D. 2009, ApJ, 690, 1772

Poleski, R., Skowron, J., Udalski, A., et al. 2014, ApJ, 795, 42

Pollack, J. B., Hubickyj, O., Bodenheimer, P., et al. 1996, Icar, 124, 62

Rhie, S. H., Becker, A. C., Bennett, D. P., et al. 1999, ApJ, 522, 1037

Schechter, P. L., Mateo, M., \& Saha, A. 1993, PASP, 105, 1342

Skowron, J., Udalski, A., Szymański, M. K., et al. 2014, ApJ, 785, 156
Spergel, D., Gehrels, N., Baltay, C., et al. 2015, arXiv:1503.03757

Sumi, T., Bennett, D. P., Bond, I. A., et al. 2010, ApJ, 710, 1641

Suzuki, D., Bennett, D. P., Sumi, T., et al. 2016, ApJ, 833, 145

Szymański, M. K., Udalski, A., Soszyński, I., et al. 2011, AcA, 61, 83

Tang, J., Bressan, A., Rosenfield, P., et al. 2014, MNRAS, 445, 4287

Thommes, E. W., Matsumura, S., \& Rasio, F. A. 2008, Sci, 321, 814

Tomaney, A. B., \& Crotts, A. P. S. 1996, AJ, 112, 2872

Twicken, J. D., Jenkins, J. M., Seader, S. E., et al. 2016, AJ, 152, 158

Udalski, A. 2003, AcA, 53, 291

Udalski, A., Jung, Y. K., Han, C., et al. 2015a, ApJ, 812, 47

Udalski, A., Szymański, M., Kałużny, J., et al. 1994, AcA, 44, 227

Udalski, A., Szymański, M. K., \& Szymański, G. 2015b, AcA, 65, 1

Wright, J. T., \& Gaudi, B. S. 2013, in Planets, Stars and Stellar Systems: Solar and Stellar Planetary Systems, Vol. 3 (Dordrecht: Springer), 489 